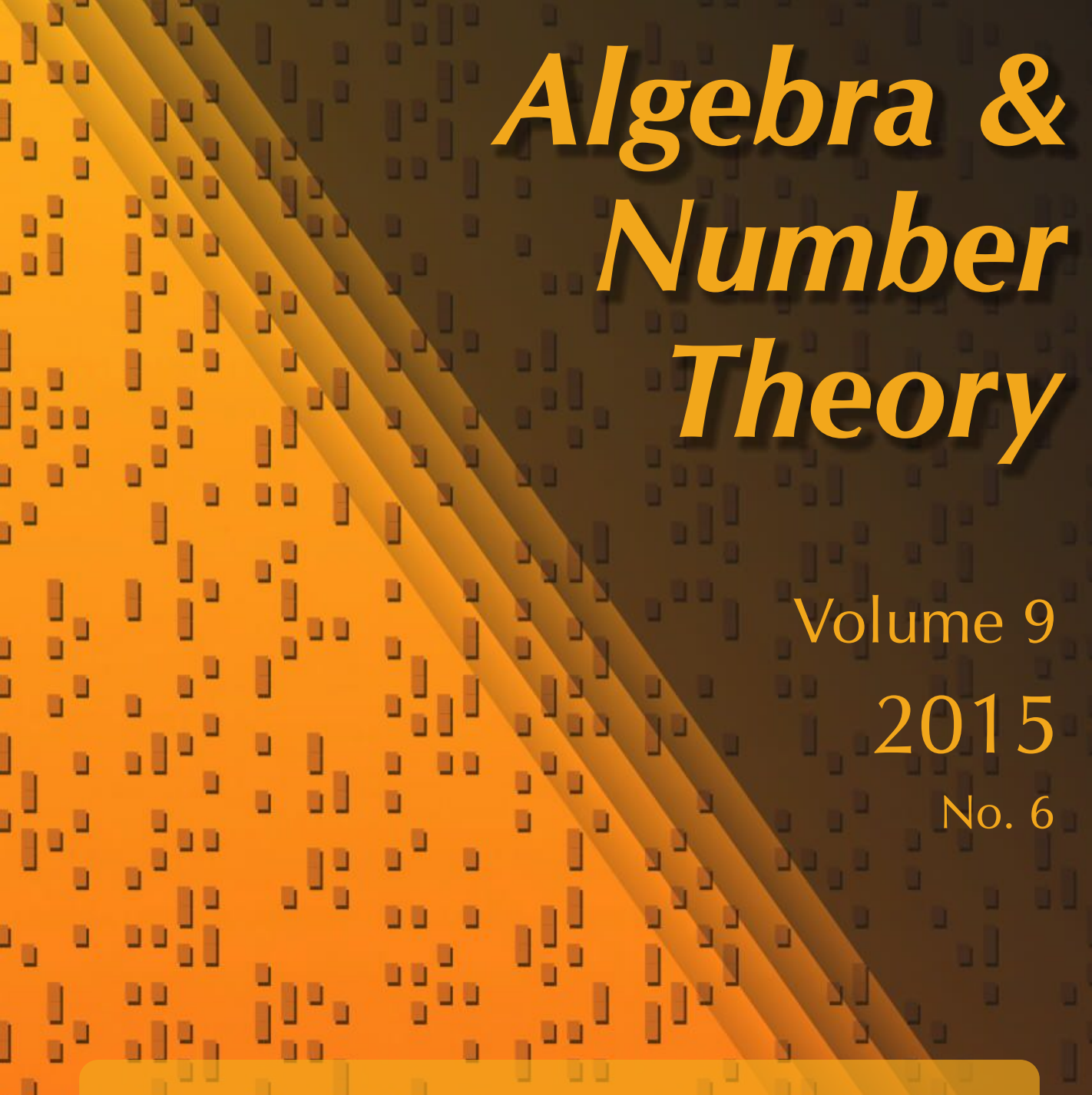

The Kac-Wakimoto character formula for the general linear Lie superalgebra

Michael Chmutov, Crystal Hoyt and Shifra Reif

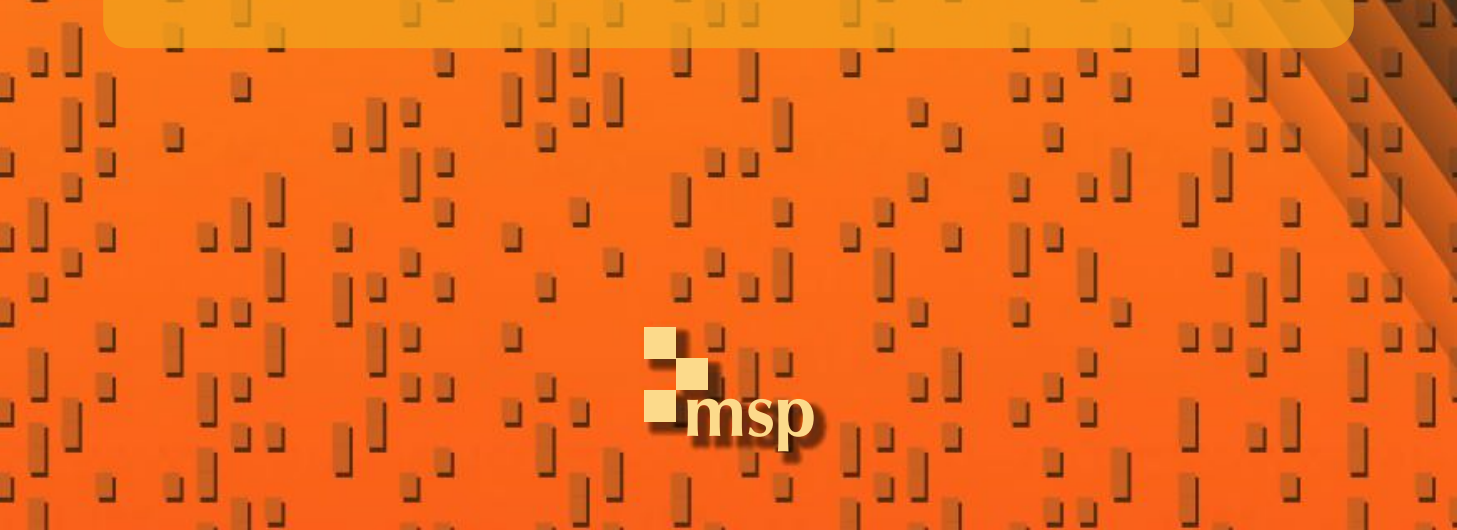




\title{
The Kac-Wakimoto character formula for the general linear Lie superalgebra
}

\author{
Michael Chmutov, Crystal Hoyt and Shifra Reif
}

\begin{abstract}
We prove the Kac-Wakimoto character formula for the general linear Lie superalgebra $\mathfrak{g l}(m \mid n)$, which was conjectured by Kac and Wakimoto in 1994. This formula specializes to the well-known Kac-Weyl character formula when the modules are typical and to the Weyl denominator identity when the module is trivial. We also prove a determinantal character formula for $\mathrm{KW}$-modules.

In our proof, we demonstrate how to use odd reflections to move character formulas between the different sets of simple roots of a Lie superalgebra. As a consequence, we show that KW-modules are precisely Kostant modules, which were studied by Brundan and Stroppel, thus yielding a simple combinatorial defining condition for KW-modules and a classification of these modules.
\end{abstract}

\section{Introduction}

It is well known that character theory for Lie superalgebras is a nontrivial generalization of the classical theory. The search for a Kac-Weyl-type character formula has been a driving force in the field. The heart of the problem lies in the existence of the so-called "atypical roots". In their absence, a formula similar to the classical Weyl character formula was proven for finite-dimensional typical highest weight modules by Kac [1977a; 1977b; 1978]. For the singly atypical weights (those with only one atypical root), a closed formula was proven by Bernstein and Leites [1980] for $\mathfrak{g l}(m \mid n)$ using geometrical methods. It was a long-standing question to generalize this formula, and many people contributed, including Van der Jeugt, Hughes, King and Thierry-Mieg [Van der Jeugt et al. 1990], who proposed a conjectural character formula for $\mathfrak{g l}(m \mid n)$, which was later proven by Su and Zhang [2007].

Serganova [1996; 1998] introduced the generalized Kazhdan-Lusztig polynomials for $\mathfrak{g l}(m \mid n)$, and used them to give a general character formula for finitedimensional irreducible representations of $\mathfrak{g l}(m \mid n)$. For each $\lambda$ and $\mu$ dominant

Hoyt was supported in part by an Aly Kaufman Fellowship at the Technion. Reif was partially supported by NSF RTG grant DMS 0943832.

MSC2010: primary 17B10; secondary 17B20, 22E47.

Keywords: character formulas, Kazhdan-Lusztig polynomials, Lie superalgebras, tame modules. 
integral weights, evaluating the Kazhdan-Lusztig polynomial $K_{\lambda, \mu}(q)$ at $q=-1$ was shown to yield the multiplicity of the Kac module $\bar{L}(\mu)$ inside the finitedimensional simple module $L(\lambda)$.

Brundan [2003] gave a new algorithm for computing the generalized KazhdanLusztig polynomials for $\mathfrak{g l}(m \mid n)$. This algorithm was proven to be combinatorially equivalent to Serganova's original algorithm by Musson and Serganova [2011]. Cheng, Wang and Zhang [Cheng et al. 2008b] showed that the generalized KazhdanLusztig polynomials for $\mathfrak{g l}(m \mid n)$ correspond to the usual parabolic Kazhdan-Lusztig polynomials of type $A$.

Su and Zhang [2007, 4.43] used Brundan's algorithm to compute the generalized Kazhdan-Lusztig polynomials and to prove a Kac-Weyl type character formula for finite-dimensional simple modules of $\mathfrak{g l}(m \mid n)$ in the standard choice of simple roots, which we refer to as the Su-Zhang character formula. When the highest weight $\lambda$ is "totally connected" (see Definition 18), every nonzero Kazhdan-Lusztig polynomial is a monomial with coefficient 1 , which drastically simplifies the $\mathrm{Su}$-Zhang character formula (4-1) [2007, 4.46].

We use the Su-Zhang character formula to prove the Kac-Wakimoto character formula for $\mathfrak{g l}(m \mid n)$, which was conjectured in [Kac and Wakimoto 1994, Theorem 3.1]. This conjecture was recently restated in [Kac and Wakimoto 2014, Conjecture 3.6], where the affine version of the formula was shown to yield a new class of modular invariant functions.

Theorem 1. Let $L$ be a finite-dimensional simple module. For any choice of simple roots $\pi$ and weight $\lambda$ such that $L=L_{\pi}(\lambda)$ and $\pi$ contains a $\lambda+\rho$-maximal isotropic subset $S_{\lambda}$, we have

$$
e^{\rho} R \cdot \operatorname{ch} L_{\pi}(\lambda)=\frac{1}{r !} \sum_{w \in W}(-1)^{l(w)} w\left(\frac{e^{\lambda+\rho}}{\prod_{\beta \in S_{\lambda}}\left(1+e^{-\beta}\right)}\right),
$$

where $r=\left|S_{\lambda}\right|$.

We call a finite-dimensional simple module $L$ a $K W$-module if there exists a set of simple roots $\pi$ that satisfies the hypothesis of Theorem 1, and we call such a $\pi$ an admissible choice of simple roots for $L$.

Our proof goes as follows. We prove that a finite-dimensional simple module is a KW-module if and only if its highest weight with respect to the standard choice of simple roots is totally connected (Theorem 21), by presenting an algorithm to move between these different bases. We generalize the arc diagrams defined in [Gorelik et al. 2012], and we use these diagrams to define the steps of our algorithm, which are composed of a specified sequence of odd reflections. We show that each step of the algorithm preserves a generalized version of the $\mathrm{Su}$-Zhang character formula, and that this generalized formula specializes to the Kac-Wakimoto character formula 
when the set of simple roots is admissible, thus proving that $\mathrm{KW}$-modules for $\mathfrak{g l}(m \mid n)$ are tame.

In this way, we obtain a character formula for each admissible set of simple roots $\pi$ and $(\lambda+\rho)$-maximal isotropic subset $S_{\lambda} \subset \pi$. We also obtain character formulas for the sets of simple roots encountered when applying the "shortening algorithm" to a totally connected weight, but not for all sets of simple roots. When the module is trivial, these character formulas specialize to the denominator identities obtained by Gorelik, Kac, Möseneder Frajria and Papi [Gorelik 2012; Gorelik et al. 2012].

We use the Kac-Wakimoto character formula to prove a determinantal character formula for $\mathrm{KW}$-modules (Theorem 44), which is motivated by the determinantal character formula proven in [Moens and Van der Jeugt 2004] for critical modules labeled by nonintersecting composite partitions. Our determinantal character formula can be expressed using the data of the "special arc diagram" for a KW-module $L$ (see Definition 24), and is useful for computer computations.

To make the paper self-contained, we give a simplified version of the proof of the $\mathrm{Su}$-Zhang character formula in the special case that the highest weight of the module is totally connected, i.e., a KW-module. Along the way, we obtain another characterization of KW-modules in terms of their Kazhdan-Lusztig polynomials (see Corollary 31), and we obtain the character formula of Theorem 37, which is motivated by the denominator identity given in [Gorelik et al. 2012, (1.10)] for the standard choice of simple roots.

An important class of KW-modules are the covariant modules. The tensor module $T(V)$ of the $(m+n)$-dimensional natural representation $V$ of $\mathfrak{g l}(m \mid n)$ is completely reducible, and its irreducible components are called covariant modules. These modules and their characters (super-Schur functions) were studied by Berele and Regev [1987], and Sergeev [1984], who in particular gave a necessary and sufficient condition on a weight $\lambda$ in terms of Young diagrams to be the highest weight of a covariant module for $\mathfrak{g l}(m \mid n)$ (with respect to the standard set of simple roots). Specifically, the irreducible components of the $k$-th tensor power of $V$ are parametrized by Young diagrams of size $k$ contained in the $(m, n)$-hook. Using this description, it is easy to show that the highest weight of a covariant module is totally connected (see Example 20). Hence, it follows from Theorem 21 that covariant modules are KW-modules, which was originally proven in [Moens and Van der Jeugt 2003].

The KW-modules studied in this paper turn out to be the same as what are called Kostant modules in [Brundan and Stroppel 2010; Brundan and Stroppel 2012] (see Remark 33). Brundan and Stroppel proved that all Kostant modules possess a BGG-type resolution, generalizing the BGG-type resolutions for covariant modules previously constructed by Cheng, Kwon and Lam [Cheng et al. 2008a]. In this way, the character formula in (4-4) can be realized as the Euler characteristic of this resolution. 


\section{Preliminaries}

2.1. The general linear Lie superalgebra. In this paper, $\mathfrak{g}$ will always denote the general linear Lie superalgebra $\mathfrak{g l}(m \mid n)$ over the complex field $\mathbb{C}$. As a vector space, $\mathfrak{g}$ can be identified with the endomorphism algebra $\operatorname{End}\left(V_{\overline{0}} \oplus V_{\overline{1}}\right)$ of a $\mathbb{Z}_{2}$-graded vector space $V_{\overline{0}} \oplus V_{\overline{1}}$ with $\operatorname{dim} V_{\overline{0}}=m$ and $\operatorname{dim} V_{\overline{1}}=n$. Then $\mathfrak{g}=\mathfrak{g}_{\overline{0}} \oplus \mathfrak{g}_{\overline{1}}$, where

$$
\mathfrak{g}_{\overline{0}}=\operatorname{End}\left(V_{\overline{0}}\right) \oplus \operatorname{End}\left(V_{\overline{1}}\right) \quad \text { and } \quad \mathfrak{g}_{\overline{1}}=\operatorname{Hom}\left(V_{\overline{0}}, V_{\overline{1}}\right) \oplus \operatorname{Hom}\left(V_{\overline{1}}, V_{\overline{0}}\right) .
$$

A homogeneous element $x \in \mathfrak{g}_{\overline{0}}$ has degree 0 , denoted $\operatorname{deg}(x)=0$, while $x \in \mathfrak{g}_{\overline{1}}$ has degree 1 , denoted $\operatorname{deg}(x)=1$. We define a bilinear operation on $\mathfrak{g}$ by letting

$$
[x, y]=x y-(-1)^{\operatorname{deg}(x) \operatorname{deg}(y)} y x
$$

on homogeneous elements and then extending linearly to all of $\mathfrak{g}$.

By fixing a basis of $V_{\overline{0}}$ and $V_{\overline{1}}$, we can realize $\mathfrak{g}$ as the set of $(m+n) \times(m+n)$ matrices, where

$$
\begin{aligned}
& \mathfrak{g}_{0}=\left\{\left(\begin{array}{cc}
A & 0 \\
0 & B
\end{array}\right) \mid A \in M_{m, m}, B \in M_{n, n}\right\}, \\
& \mathfrak{g}_{\overline{1}}=\left\{\left(\begin{array}{cc}
0 & C \\
D & 0
\end{array}\right) \mid C \in M_{m, n}, D \in M_{n, m}\right\},
\end{aligned}
$$

and $M_{r, s}$ denotes the set of $r \times s$ matrices.

The Cartan subalgebra $\mathfrak{h}$ of $\mathfrak{g}$ is the set of diagonal matrices, and it has a natural basis

$$
\left\{E_{1,1}, \ldots, E_{m, m} ; E_{m+1, m+1}, \ldots, E_{m+n, m+n}\right\},
$$

where $E_{i j}$ denotes the matrix whose $i j$-entry is 1 and there are 0 s elsewhere. Fix the dual basis $\left\{\varepsilon_{1}, \ldots, \varepsilon_{m} ; \delta_{1}, \ldots, \delta_{n}\right\}$ for $\mathfrak{h}^{*}$. We define a bilinear form on $\mathfrak{h}^{*}$ by $\left(\varepsilon_{i}, \varepsilon_{j}\right)=\delta_{i j}=-\left(\delta_{i}, \delta_{j}\right)$ and $\left(\varepsilon_{i}, \delta_{j}\right)=0$, and use it to identify $\mathfrak{h}$ with $\mathfrak{h}^{*}$.

Then $\mathfrak{g}$ has a root space decomposition $\mathfrak{g}=\mathfrak{h} \oplus\left(\bigoplus_{\alpha \in \Delta_{\overline{0}}} \mathfrak{g}_{\alpha}\right) \oplus\left(\bigoplus_{\alpha \in \Delta_{\overline{\mathrm{l}}}} \mathfrak{g}_{\alpha}\right)$, where the set of roots of $\mathfrak{g}$ is $\Delta=\Delta_{\overline{0}} \cup \Delta_{\overline{1}}$, with

$$
\begin{aligned}
& \Delta_{\overline{0}}=\left\{\varepsilon_{i}-\varepsilon_{j} \mid 1 \leq i \neq j \leq m\right\} \cup\left\{\delta_{k}-\delta_{l} \mid 1 \leq k \neq l \leq n\right\}, \\
& \Delta_{\overline{1}}=\left\{ \pm\left(\varepsilon_{i}-\delta_{k}\right) \mid 1 \leq i \leq m, 1 \leq k \leq n\right\},
\end{aligned}
$$

and

$$
\mathfrak{g}_{\varepsilon_{i}-\varepsilon_{j}}=\mathbb{C} E_{i j}, \quad \mathfrak{g}_{\delta_{k}-\delta_{l}}=\mathbb{C} E_{m+k, m+l}, \quad \mathfrak{g}_{\varepsilon_{i}-\delta_{k}}=\mathbb{C} E_{i, m+k}, \quad \mathfrak{g}_{\delta_{k}-\varepsilon_{i}}=\mathbb{C} E_{m+k, i} .
$$

A set of simple roots $\pi \subset \Delta$ determines a decomposition of $\Delta$ into positive and negative roots $\Delta=\Delta^{+} \cup \Delta^{-}$. There is a corresponding triangular decomposition of $\mathfrak{g}$ given by $\mathfrak{g}=\mathfrak{n}^{+} \oplus \mathfrak{h} \oplus \mathfrak{n}^{-}$, where $\mathfrak{n}^{ \pm}=\bigoplus_{\alpha \in \Delta^{ \pm}} \mathfrak{g}_{\alpha}$. Let $\Delta_{\bar{d}}^{+}=\Delta_{\bar{d}} \cap \Delta^{+}$for $d \in\{0,1\}$, and define $\rho_{\pi}=\frac{1}{2} \sum_{\alpha \in \Delta_{\overline{0}}^{+}} \alpha-\frac{1}{2} \sum_{\alpha \in \Delta_{\overline{1}}^{+}} \alpha$. Then for $\alpha \in \pi$ we have $\left(\rho_{\pi}, \alpha\right)=(\alpha, \alpha) / 2$. 
The Weyl group of $\mathfrak{g}$ is $W=\operatorname{Sym}_{m} \times \operatorname{Sym}_{n}$, and $W$ acts on $\mathfrak{h}^{*}$ by permuting separately indices on $\varepsilon$ and $\delta$. In particular, the even reflection $s_{\varepsilon_{i}-\varepsilon_{j}}$ interchanges indices $i$ and $j$ on $\varepsilon$ and fixes all the others, while $s_{\delta_{k}-\delta_{l}}$ interchanges indices $k$ and $l$ on $\delta$ and fixes all the others.

A proof of the following lemma can be found in [Gorelik 2012, 4.1.1]:

Lemma 2. For any $\mu \in \mathfrak{h}_{\mathbb{R}}^{*}$ the stabilizer of $\mu$ in $W$ is either trivial or contains a reflection.

Suppose $\beta \in \pi$ is an odd (isotropic) root. An odd reflection $r_{\beta}$ of each $\alpha \in \pi$ is defined by

$$
r_{\beta}(\alpha)= \begin{cases}c l-\alpha & \text { if } \beta=\alpha, \\ \alpha & \text { if }(\alpha, \beta)=0, \\ \alpha+\beta & \text { if }(\alpha, \beta) \neq 0 .\end{cases}
$$

Then $r_{\beta} \pi:=\left\{r_{\beta}(\alpha) \mid \alpha \in \pi\right\}$ is also a set of simple roots for $\mathfrak{g}$ [Serganova 2011]. The corresponding root decomposition is $\Delta=\Delta^{\prime+} \cup \Delta^{\prime-}$, where $\Delta_{\overline{0}}^{\prime+}=\Delta_{\overline{0}}^{+}$is unchanged and $\Delta_{\overline{1}}^{\prime+}=\left(\Delta_{\overline{1}}^{+} \backslash\{\beta\}\right) \cup\{-\beta\}$. Using a sequence of even and odd reflections, one can move between any two sets of simple roots for $\mathfrak{g}$. Moreover, if $\pi$ and $\pi^{\prime \prime}$ have the property that $\Delta^{\prime \prime}{ }_{0}^{+}=\Delta_{\overline{0}}^{+}$, then there exists a sequence of odd reflections from $\pi$ to $\pi^{\prime \prime}$.

We denote by $\pi_{\mathrm{st}}$ the standard choice of simple roots

$$
\pi_{\mathrm{st}}=\left\{\varepsilon_{1}-\varepsilon_{2}, \ldots, \varepsilon_{m-1}-\varepsilon_{m}, \varepsilon_{m}-\delta_{1}, \delta_{1}-\delta_{2} \ldots, \delta_{n-1}-\delta_{n}\right\} .
$$

The corresponding decomposition $\Delta=\Delta^{+} \cup \Delta^{-}$is given by

$$
\Delta_{\overline{0}}^{+}=\left\{\varepsilon_{i}-\varepsilon_{j}\right\}_{1 \leq i<j \leq m} \cup\left\{\delta_{k}-\delta_{l}\right\}_{1 \leq k<l \leq n} \text { and } \Delta_{\overline{1}}^{+}=\left\{\varepsilon_{i}-\delta_{k}\right\}_{1 \leq i \leq m, 1 \leq k \leq n} .
$$

The standard choice $\pi_{\mathrm{st}}$ has the unique property that $W$ fixes $\Delta_{\overline{1}}^{+}$. Moreover, $\pi_{\mathrm{st}}$ contains a basis for $\Delta_{\overline{0}}^{+}$, which is denoted by $\pi_{0}^{-}:=\pi_{\mathrm{st}} \cap \Delta_{\overline{0}}^{+}$.

The root lattice $Q=\sum_{\alpha \in \pi} \mathbb{Z} \alpha$ is independent of the choice of $\pi$. Let $Q_{\pi}^{+}=$ $\sum_{\alpha \in \pi} \mathbb{N} \alpha$, where $\mathbb{N}=\{0,1,2, \ldots\}$, and define a partial order on $\mathfrak{h}^{*}$ by $\mu>v$ when $\mu-v \in Q_{\text {st }}^{+}$.

Remark 3. For convenience, we fix $\Delta_{\overline{0}}^{+}$as in (2-1). This choice is arbitrary since we can relabel the indices of the $\varepsilon$ 's and $\delta$ 's. We let $l(w)$ denote the length of $w \in W$ with respect to the set of simple reflections $s_{\varepsilon_{1}-\varepsilon_{2}}, \ldots, s_{\varepsilon_{m-1}-\varepsilon_{m}} ; s_{\delta_{1}-\delta_{2}}, \ldots, s_{\delta_{n-1}-\delta_{n}}$ generating $W$.

2.2. Finite-dimensional modules for $\mathfrak{g l}(\boldsymbol{m} \mid \boldsymbol{n})$. For each set of simple roots $\pi$ and weight $\lambda \in \mathfrak{h}^{*}$, the Verma module of highest weight $\lambda$ is the induced module

$$
M_{\pi}(\lambda):=\operatorname{Ind}_{\mathfrak{n}^{+} \oplus \mathfrak{h}}^{\mathfrak{g}} \mathbb{C}_{\lambda},
$$


where $\mathbb{C}_{\lambda}$ is the one-dimensional module such that $h \in \mathfrak{h}$ acts by scalar multiplication of $\lambda(h)$ and $\mathfrak{n}^{+}$acts trivially. The Verma module $M_{\pi}(\lambda)$ has a unique simple quotient, which we denote by $L_{\pi}(\lambda)$ or simply by $L(\lambda)$. Given $\pi$ and $\lambda$, we define

$$
\lambda_{\pi}^{\rho}:=\lambda+\rho_{\pi},
$$

which we also denote by $\lambda^{\rho}$. If $L_{\pi}(\lambda) \cong L_{\pi^{\prime}}\left(\lambda^{\prime}\right)$ for some $\lambda, \lambda^{\prime} \in \mathfrak{h}^{*}$ and $\pi^{\prime}=r_{\beta} \pi$ for an odd reflection $r_{\beta}$ with $\beta \in \pi$, then

$$
\left(\lambda^{\prime}\right)^{\rho}= \begin{cases}\lambda^{\rho} & \text { if }(\lambda, \beta) \neq 0 \\ \lambda^{\rho}+\beta & \text { if }(\lambda, \beta)=0\end{cases}
$$

For each $\lambda \in \mathfrak{h}^{*}$, let $L_{\overline{0}}(\lambda)$ denote the simple highest weight $\mathfrak{g}_{\overline{0}}$-module with respect to $\pi_{\overline{0}}$. The Kac module of highest weight $\lambda$ with respect to $\pi_{\mathrm{st}}$ is the induced module

$$
\bar{L}(\lambda):=\operatorname{Ind}_{\mathfrak{g}_{0} \oplus \mathfrak{n}_{\overline{1}}^{+}}^{\mathfrak{g}} L_{\overline{0}}(\lambda)
$$

defined by letting $\mathfrak{n}_{\overline{1}}^{+}:=\bigoplus_{\alpha \in \Delta_{\overline{1}}^{+}} \mathfrak{g}_{\alpha}$ act trivially on the $\mathfrak{g}_{0}$-module $L_{\overline{0}}(\lambda)$. The unique simple quotient of $\bar{L}(\lambda)$ is $L_{\pi_{\mathrm{st}}}(\lambda)$.

A weight $\lambda \in \mathfrak{h}^{*}$ is called dominant if $2(\lambda, \alpha) /(\alpha, \alpha) \geq 0$ for all $\alpha \in \Delta_{\overline{0}}^{+}$, strictly dominant if $2(\lambda, \alpha) /(\alpha, \alpha)>0$ for all $\alpha \in \Delta_{\overline{0}}^{+}$and integral if $2(\lambda, \alpha) /(\alpha, \alpha) \in \mathbb{Z}$ for all $\alpha \in \Delta_{\overline{0}}^{+}$. It is sufficient to check dominance and integrality on the set $\pi_{\overline{0}}$ of simple roots of $\Delta_{\overline{0}}^{+}$.

The proof of the following lemma is straightforward when viewing $L_{\pi}(\lambda)$ as a $\mathfrak{g}_{0}$-module.

Lemma 4. If the simple module $L_{\pi}(\lambda)$ is finite-dimensional, then $\lambda$ is a dominant integral weight.

For a proof of the following proposition, see for example [Musson 2012, 14.1.1]:

Proposition 5. For $\mathfrak{g}=\mathfrak{g l}(m \mid n)$ and $\lambda \in \mathfrak{h}^{*}$, the following are equivalent:

(1) The simple highest weight $\mathfrak{g}$-module $L_{\pi_{\mathrm{st}}}(\lambda)$ is finite-dimensional.

(2) The Kac module $\bar{L}(\lambda)$ is finite-dimensional.

(3) The simple highest weight $\mathfrak{g}_{0}^{-}$-module $L_{\overline{0}}(\lambda)$ is finite-dimensional.

(4) $\lambda$ is a dominant integral weight.

(5) $\lambda_{\mathrm{st}}^{\rho}$ is a strictly dominant integral weight.

2.3. Atypical modules. The atypicality of $L_{\pi}(\lambda)$ is the maximal number of linearly independent positive roots $\beta_{1}, \ldots, \beta_{r}$ such that $\left(\beta_{i}, \beta_{j}\right)=0$ and $\left(\lambda^{\rho}, \beta_{i}\right)=0$ for $i, j=1, \ldots, r$. Such a set $S=\left\{\beta_{1}, \ldots, \beta_{r}\right\}$ is called a $\lambda^{\rho}$-maximal isotropic set. The module $L_{\pi}(\lambda)$ is called typical if this set is empty, and atypical otherwise. The atypicality of a simple finite-dimensional module is independent of the choice of simple roots. 
Definition 6. We call a simple finite-dimensional module $L$ a $K W$-module if $L \cong$ $L_{\pi}(\lambda)$ for some choice of simple roots $\pi$ which contains a $\lambda^{\rho}$-maximal isotropic set $S \subset \pi$. In this case, we call such a $\pi$ an admissible choice of simple roots for $L$.

Remark 7. A typical module is a KW-module with $S=\varnothing$.

Let $P$ denote the set of integral weights, $P^{+}$the set of dominant integral weights, and define

$$
\mathbb{P}^{+}=\left\{\mu \in P^{+} \mid\left(\mu_{\pi}^{\rho}, \varepsilon_{i}\right) \in \mathbb{Z},\left(\mu_{\pi}^{\rho}, \delta_{j}\right) \in \mathbb{Z}\right\} .
$$

Note that the definition of $\mathbb{P}^{+}$is independent of the choice of $\pi$, since changing the set of simple roots by an odd reflection only changes the entries of $\lambda_{\pi}^{\rho}$ by integer values.

Remark 8. When studying the character of a simple finite-dimensional atypical module, we may restrict to the case that $\lambda \in \mathbb{P}^{+}$. Indeed, let $\lambda \in P^{+}$; then the module $L_{\pi}(\lambda)$ is atypical if and only if $\left(\lambda_{\pi}^{\rho}, \varepsilon_{i}\right)=\left(\lambda_{\pi}^{\rho}, \delta_{j}\right)$ for some $\left(\varepsilon_{i}-\delta_{j}\right) \in \Delta_{\overline{1}}$. So, by tensoring $L_{\pi}(\lambda)$ with a one-dimensional module with character $e^{c\left(\sum_{i=1}^{m} \varepsilon_{i}-\sum_{j=1}^{n} \delta_{j}\right)}$ for appropriate $c \in \mathbb{C}$, we obtain a module $L_{\pi}\left(\lambda^{\prime}\right)$ with $\lambda^{\prime} \in \mathbb{P}^{+}$.

Fix a set of simple roots $\pi$, a weight $\lambda \in \mathbb{P}^{+}$and a $\lambda^{\rho}$-maximal isotropic set $S_{\lambda} \subset \Delta_{\overline{1}}^{+}$. Write

$$
\lambda_{\pi}^{\rho}=\sum_{i=1}^{m} a_{i} \varepsilon_{i}-\sum_{j=1}^{n} b_{j} \delta_{j} .
$$

We refer to the coefficients $a_{i}$ and $b_{j}$ as the $\varepsilon_{i}$-entry and $\delta_{j}$-entry, respectively. If one of $\pm\left(\varepsilon_{k}-\delta_{l}\right)$ is in $S_{\lambda}$, then we call the $\varepsilon_{k}$ - and $\delta_{l}$-entries atypical. Otherwise, an entry is called typical.

We denote by $\left(\lambda_{\pi}^{\rho}\right)^{\Uparrow}$ the element obtained from $\lambda_{\pi}^{\rho}$ by replacing all its atypical entries by the maximal atypical entry. Note that this can depend on the choice of $S_{\lambda}$, and not only on $\lambda_{\pi}^{\rho}$. However, for $\pi_{\text {st }}$ and $\lambda \in \mathbb{P}^{+}$, there is a unique $S_{\lambda} \subset \Delta^{+}$. If $v \in \mathfrak{h}^{*}$ can be written as $v=\sum_{\alpha \in S_{\lambda}} k_{\alpha} \alpha$, then we define

$$
|v|_{S_{\lambda}}:=\sum_{\alpha \in S_{\lambda}} k_{\alpha}
$$

Observe that $\left|\left(\lambda_{\pi}^{\rho}\right)^{\Uparrow}-\lambda_{\pi}^{\rho}\right|_{S_{\lambda}}$ is nonnegative integer.

2.4. Arc diagrams. We generalize the arc diagrams defined in [Gorelik et al. 2012]. Let $L$ be a finite-dimensional atypical module. For each set of simple roots $\pi$, weight $\lambda \in \mathbb{P}^{+}$such that $L=L_{\pi}(\lambda)$ and $\lambda^{\rho}$-maximal isotropic set $S_{\lambda} \subset \Delta_{\overline{1}}^{+}$, there is an arc diagram that encodes the data $\pi, \lambda_{\pi}^{\rho}$ and $S_{\lambda}$. 
In order to define the arc diagram corresponding to the data $\left(\pi, \lambda_{\pi}^{\rho}, S_{\lambda}\right)$, we first define a total order on the set $\left\{\varepsilon_{1}, \ldots, \varepsilon_{m}\right\} \cup\left\{\delta_{1}, \ldots, \delta_{n}\right\}$ determined by $\Delta^{+}$. In particular, $\varepsilon_{i}<\varepsilon_{i+1}, \delta_{j}<\delta_{j+1}$, and for each $i$ and $j$ we let

$$
\begin{array}{cc}
\delta_{j}<\varepsilon_{i} & \text { if }\left(\delta_{j}-\varepsilon_{i}\right) \in \Delta^{+}, \\
\varepsilon_{i}<\delta_{j} & \text { if }\left(\varepsilon_{i}-\delta_{j}\right) \in \Delta^{+} .
\end{array}
$$

Let $T=\left\{\gamma_{1}, \ldots, \gamma_{m+n}\right\}$ be this totally ordered set, and express $\lambda_{\pi}^{\rho}$ as in (2-2). The nodes and entries of the diagram are determined from left to right by the elements $\gamma_{k} \in T, k=1, \ldots, m+n$ by putting a node $\bullet$ labeled with the entry $a_{i}$ if $\gamma_{k}=\varepsilon_{i}$ and a node $\times$ labeled with the entry $b_{j}$ if $\gamma_{k}=\delta_{j}$. The set $S_{\lambda}$ determines an arc arrangement as follows: The $\varepsilon_{i}$-node $\bullet$ and the $\delta_{j}$-node $\times$ are connected by an arc when one of $\pm\left(\varepsilon_{i}-\delta_{j}\right)$ is in $S_{\lambda}$, and in this case $a_{i}=b_{j}$. A node and its entry are called atypical if the node is connected by some arc, and typical otherwise.

\section{Example 9. Suppose}

$$
\begin{aligned}
\pi & =\left\{\varepsilon_{1}-\varepsilon_{2}, \varepsilon_{2}-\delta_{1}, \delta_{1}-\varepsilon_{3}, \varepsilon_{3}-\delta_{2}, \delta_{2}-\delta_{3}, \delta_{3}-\varepsilon_{4}\right\}, \\
\lambda_{\pi}^{\rho} & =7 \varepsilon_{1}+5 \varepsilon_{2}+5 \varepsilon_{3}+2 \varepsilon_{4}-5 \delta_{1}-6 \delta_{2}-7 \delta_{3}, \\
S_{\lambda} & =\left\{\varepsilon_{1}-\delta_{3}, \delta_{1}-\varepsilon_{3}\right\} .
\end{aligned}
$$

Then the corresponding arc diagram will be:

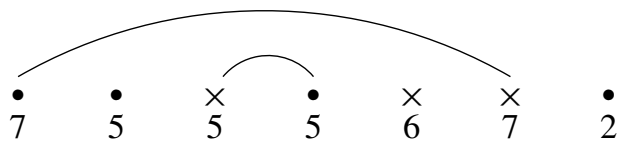

We can recover the data $\left(\pi, \lambda_{\pi}^{\rho}, S_{\lambda}\right)$ from an arc diagram as follows. Label the $\bullet$-nodes from left to right by $\varepsilon_{1}, \ldots, \varepsilon_{m}$, and the $\times$-nodes by $\delta_{1}, \ldots, \delta_{n}$. Let $T=\left\{\gamma_{1}, \ldots, \gamma_{m+n}\right\}$ be the ordered set determined by this labeling. Then $\pi=$ $\left\{\gamma_{1}-\gamma_{2}, \gamma_{2}-\gamma_{3}, \ldots, \gamma_{m+n-1}-\gamma_{m+n}\right\}, \lambda_{\pi}^{\rho}$ is given by (2-2), where $a_{i}$ is the $\varepsilon_{i}$-entry and $b_{j}$ is the $\delta_{j}$-entry, and $S_{\lambda}=\left\{\gamma_{i}-\gamma_{j} \mid i<j\right.$ and $\gamma_{i}$ is connected by an arc to $\left.\gamma_{j}\right\}$.

Remark 10. All entries of an arc diagram are integers, and adjacent $\bullet$-entries are strictly decreasing, while adjacent $\times$-entries are strictly increasing, because $\lambda \in \mathbb{P}^{+}$ and $\lambda_{\pi}^{\rho}=\lambda+\rho_{\pi}$ (see Remark 8).

Remark 11. We call the arc diagram for the standard choice of simple roots the standard arc diagram. For the standard arc diagram, there is only one possible arc arrangement and all arcs are "nested". See for example diagram (3-3).

Since $S_{\lambda} \subset \Delta_{\overline{1}}^{+}$, an arc is always connected to a $\bullet$-node and a $\times$-node. We call an arc short if the $\bullet$-node and $\times$-node are adjacent. We say that an arc has $\bullet-\times$ type if the $\bullet$ precedes the $\times$, and $\times-\bullet$ type if the $\times$ precedes the $\bullet$. Note that no two 
arcs can share an endpoint, since $S_{\lambda}$ is a $\lambda^{\rho}$-maximal isotropic set. Moreover, an arc diagram by definition has a maximal arc arrangement, that is, it is not possible to add an arc to the diagram between typical $\bullet-$ and $\times$-nodes with equal entries.

Adjacent $\bullet-$ and $\times$-nodes correspond to an odd simple root $\beta$, and applying the odd reflection $r_{\beta}$ swaps these nodes. In terms of the diagram, this means that if $a \neq b$, then

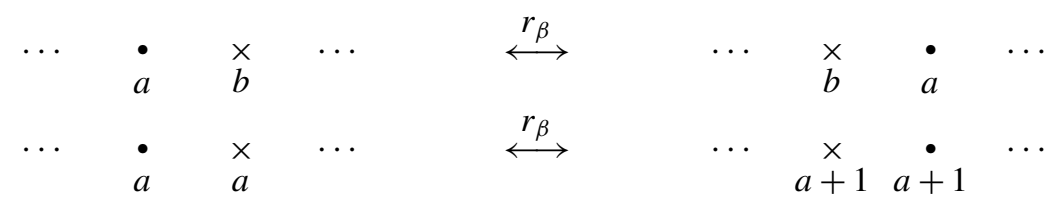

while all other nodes and entries are unchanged.

2.5. Weight diagrams. Weight diagrams are a convenient way to work with the highest weight of a module with respect to the standard choice of simple roots. They were introduced by Brundan and Stroppel [2011] and were used to give algorithmic character formulas for basic classical Lie superalgebras in [Gruson and Serganova 2010] (see also [Su and Zhang 2012, 5.1]).

Let $\lambda \in \mathbb{P}^{+}$, and write $\lambda_{\text {st }}^{\rho}$ as in (2-2). On the $\mathbb{Z}$-lattice, put $\times$ above $t$ if $t \in$ $\left\{a_{i}\right\} \cap\left\{b_{j}\right\}$, put $>$ above $t$ if $t \in\left\{a_{i}\right\} \backslash\left\{b_{j}\right\}$, and put $<$ above $t$ if $t \in\left\{b_{i}\right\} \backslash\left\{a_{j}\right\}$. If $t \notin\left\{a_{i}\right\} \cup\left\{b_{j}\right\}$, then we refer to the placeholder above $t$ as an empty spot.

Example 12. If

$$
\lambda_{\mathrm{st}}^{\rho}=10 \varepsilon_{1}+9 \varepsilon_{2}+7 \varepsilon_{3}+5 \varepsilon_{4}+4 \varepsilon_{5}-\delta_{1}-4 \delta_{2}-6 \delta_{3}-7 \delta_{4},
$$

then the corresponding weight diagram $D_{\lambda}$ is

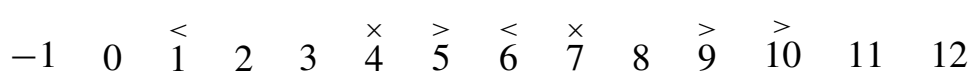

2.6. Characters for category $\mathrm{O}$. Let $M$ be a module from the BGG category 0 [Musson 2012, 8.2.3]. Then $M$ has a weight-space decomposition $M=\bigoplus_{\mu \in \mathfrak{h}^{*}} M_{\mu}$, where $M_{\mu}=\left\{x \in M \mid h . x=\mu(h) x\right.$ for all $\left.h \in \mathfrak{h}^{*}\right\}$, and the character of $M$ is by definition $\operatorname{ch} M=\sum_{\mu \in \mathfrak{h}^{*}} \operatorname{dim} M_{\mu} e^{\mu}$.

Denote by $\mathscr{E}$ the algebra of rational functions $\mathbb{Q}\left(e^{v}, v \in \mathfrak{h}^{*}\right)$. The group $W$ acts on $\mathscr{E}$ by mapping $e^{v}$ to $e^{w(v)}$. Corresponding to a choice of positive roots $\Delta^{+}$, the Weyl denominator of $\mathfrak{g}$ is defined to be

$$
R=\frac{\prod_{\alpha \in \Delta_{\overline{0}}^{+}}\left(1-e^{-\alpha}\right)}{\prod_{\alpha \in \Delta_{\overline{1}}^{+}}\left(1+e^{-\alpha}\right)} .
$$


Then $e^{\rho} R$ is $W$-skew-invariant, i.e., $w\left(e^{\rho} R\right)=(-1)^{l(w)} e^{\rho} R$, and $\operatorname{ch} L(\lambda)$ is $W$ invariant for $\lambda \in P^{+}$. The character of a Verma module $M(\lambda)$ with $\lambda \in \mathfrak{h}^{*}$ is $\operatorname{ch} M(\lambda)=e^{\lambda} R^{-1}$. The character of the Kac module $\bar{L}(\lambda)$ with $\lambda \in P^{+}$is

$$
\operatorname{ch} \bar{L}(\lambda)=\frac{1}{e^{\rho} R} \sum_{w \in W}(-1)^{l(w)} w\left(e^{\lambda^{\rho}}\right) .
$$

For $X \in \mathscr{E}$, we define

$$
\mathscr{F}_{W}(X):=\sum_{w \in W}(-1)^{l(w)} w(X) .
$$

The proof of the following lemma is immediate:

Lemma 13. Let $X \in \mathscr{E}$. If $\sigma(X)=X$ for some reflection $\sigma \in W$, then $F_{W}(X)=0$.

\section{Highest weights of KW-modules}

We describe the highest weights and arc diagrams of KW-modules with respect to different choices of simple roots.

3.1. An admissible choice of simple roots. Let us characterize the highest weight $\lambda$ of a KW-module $L$ with respect to an admissible choice of simple roots $\pi$ and a $\lambda^{\rho}$-maximal isotropic subset $S \subset \pi$.

Lemma 14. Consider two arcs in the arc diagram of $\lambda^{\rho}$ with no arcs between them. $U p$ to a reflection along these arcs, the corresponding subdiagram has one of the following forms:

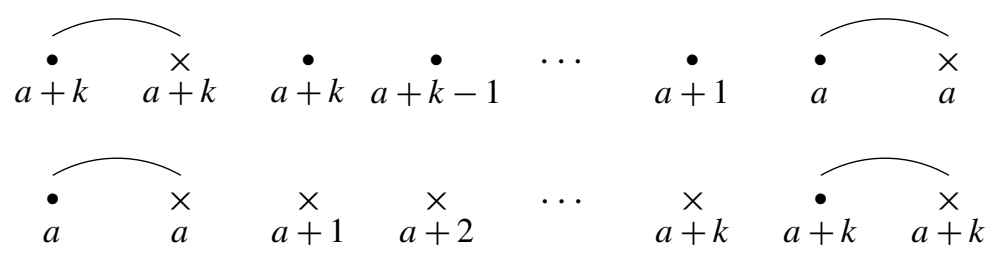

Equivalently, the neighboring $\bullet-$ and $\times$-entries are equal, and the entries between the two arcs are either all of $\bullet$-type and are increasing consecutive integers or are all of $\times$-type and are decreasing consecutive integers. Moreover, diagrams (3-1) and (3-2) cannot both be a subdiagram of the same admissible diagram.

Proof. We shall analyze the entries of $\lambda$ and $\rho$ separately.

Let us show that all the entries of $\lambda$ in the range of the subdiagram are equal. We write $\lambda=\sum_{i=1}^{m} a_{i} \varepsilon_{i}-\sum_{i=1}^{n} b_{i} \delta_{i}$ and suppose that the arcs in diagram correspond to the simple isotropic roots $\alpha=\varepsilon_{i}-\delta_{i^{\prime}}$ and $\beta=\varepsilon_{j}-\delta_{j^{\prime}}$, with $i<j, i^{\prime}<j^{\prime}$. Since $\alpha$ and $\beta$ are simple and isotropic, they are orthogonal to $\rho$ and hence to $\lambda$. This implies that $a_{i}=b_{i^{\prime}}$ and $a_{j}=b_{j^{\prime}}$. Since $\lambda$ is dominant, $a_{i} \geq a_{i+1} \geq \cdots \geq a_{j}$ and $b_{i^{\prime}} \leq b_{i^{\prime}+1} \leq \cdots \leq b_{j^{\prime}}$. Hence, $a_{i}=\cdots=a_{j}=b_{i^{\prime}}=\cdots=b_{j^{\prime}}$. 
It follows that two entries in the subdiagram are equal if and only if they are equal in $\rho$. Since $\rho$ is orthogonal to all simple isotropic roots, the $\bullet-$ and $\times-$ entries are equal when adjacent. Hence, at least one of them must be connected with an arc, since otherwise we contradict the maximality property of the arc arrangement. Therefore, the entries between the two arcs are either all of $\bullet$-type or all of $\times$-type.

The difference between two consecutive entries of the subdiagram of $\lambda^{\rho}$ will be as in $\rho$, since all such entries of $\lambda$ are equal. In particular, they should decrease (resp. increase) by 1 whenever we have consecutive $\bullet$-entries (resp. $\times$-entries).

The following definition was introduced in [Moens and Van der Jeugt 2004, Proposition 1] for the standard choice of simple roots.

Definition 15. Let $\pi$ be any set of simple roots and let $\lambda \in \mathfrak{h}^{*}$. Write $\lambda^{\rho}=$ $\sum_{i=1}^{m} a_{i} \varepsilon_{i}-\sum_{i=1}^{n} b_{i} \delta_{i}$. Suppose $S_{\lambda}=\left\{\varepsilon_{m_{i}}-\delta_{n_{i}}\right\}_{i=1}^{r}$ is a $\lambda^{\rho}$-maximal isotropic set ordered so that $a_{m_{1}} \leq a_{m_{2}} \leq \cdots \leq a_{m_{r}}$. We say that $\lambda^{\rho}$ satisfies the interval property if all the integers between $a_{m_{1}}$ and $a_{m_{r}}$ (equivalently, between $b_{n_{1}}$ and $b_{n_{r}}$ ) are contained in the set $\left\{a_{i}, b_{j}\right\}_{i=1, \ldots, m ; j=1, \ldots, n}$.

Corollary 16. Let $L$ be a $K W$-module, and let $\lambda$ be its highest weight with respect to an admissible choice of simple roots. Then $\lambda^{\rho}$ satisfies the interval property.

Remark 17. Note that the interval property is not a property of a module. In particular, if $\pi_{1}, \pi_{2}$ are two choices of simple roots and $\lambda_{1}, \lambda_{2} \in \mathfrak{h}^{*}$ are such that $=L_{\pi_{1}}\left(\lambda_{\pi_{1}}\right)=L_{\pi_{2}}\left(\lambda_{\pi_{2}}\right)$, we can have that $\lambda_{\pi_{1}}^{\rho}$ satisfies the interval property but $\lambda_{\pi_{2}}^{\rho}$ doesn't. For example,

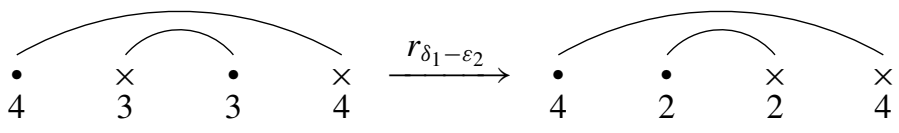

3.2. Totally connected weights in the standard choice. We prove a criterion for a module to be a KW-module given its highest weight $\lambda_{\text {st }}$ with respect to the standard choice of simple roots $\pi_{\mathrm{st}}$. The following definition is equivalent to the one given in [Su and Zhang 2007], which was first observed in [Moens and Van der Jeugt 2004].

Definition 18. Let $\lambda_{\mathrm{st}} \in \mathbb{P}^{+}$. We say that $\lambda_{\mathrm{st}}$ is totally connected if $\lambda_{\mathrm{st}}^{\rho}$ satisfies the interval property with respect to $\pi_{\mathrm{st}}$.

Remark 19. In terms of weight diagrams, this is equivalent to the condition that there are no empty spots between the $x$ 's. For example, diagram (2-4) is totally connected, whereas diagram (4-3) is not. 
Example 20. The highest weight of a covariant module is totally connected. Indeed, such a module corresponds to a partition $\mu$ of $k$ that lies in the $(m, n)$-hook, i.e., $\mu_{m+1} \leq n$ [Berele and Regev 1987; Sergeev 1984]. The corresponding covariant module is $L_{\pi_{\mathrm{st}}}(\lambda)$, where

$$
\lambda=\mu_{1} \varepsilon_{1}+\cdots+\mu_{m} \varepsilon_{m}+\tau_{1} \delta_{1}+\cdots+\tau_{n} \delta_{n},
$$

$\tau_{j}=\max \left\{0, \mu_{j}^{\prime}-m\right\}$ for $j=1, \ldots, n$, and $\mu_{j}^{\prime}$ is the length of the $j$-th column (see for example [Van der Jeugt et al. 1990, Section V]). Then $\tau_{j}=0$ for $j>\mu_{m}$ and the arc diagram of $\lambda^{\rho}$ is

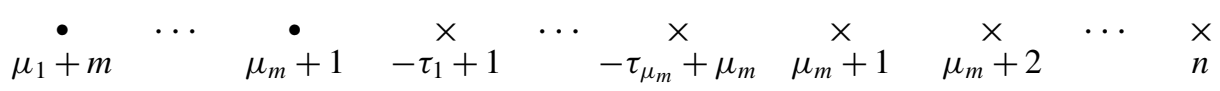

Since $\mu_{m}+1>-\tau_{\mu_{m}}+\mu_{m}$, there are no arcs connected to the first $\mu_{m} \times$-nodes. Since the rest of the $\times$-entries are consecutive integers, $\lambda^{\rho}$ satisfies the interval property and hence $\lambda$ is totally connected. The covariant module $L_{\pi_{\mathrm{st}}}(\lambda)$ is typical if and only if $\mu_{m} \geq n$.

Theorem 21. The finite-dimensional simple module $L$ is a $K W$-module if and only if its highest weight with respect to the standard choice of simple roots is totally connected.

Proof of Theorem 21 " $\Rightarrow$ ". We start with an arc diagram that corresponds to an admissible choice of simple roots, that is, all arcs are short, and we move to the standard arc diagram by applying a sequence of odd reflections which preserve the interval property. We begin with reflecting along all the arcs which are of $\times-\bullet$ type, and get another admissible choice of simple roots, for which the interval property holds by Corollary 16 .

Now we push all the $x$-entries to the right one at a time, starting with the rightmost $\times$-entry. All of our reflections are along consecutive $\times$ - and $\bullet$-entries, and at each reflection there are several cases. If the entries below the $\times$ and the are not equal, then the reflection does not change them and the interval property is clearly preserved.

If the $x$ - and - -entries are equal then at least one of them is connected to an arc, since otherwise the number of arcs could be increased, contradicting the maximality property of the arc arrangement. So there are three possibilities, namely, either the $\bullet$-entry or the $\times$-entry is connected to an arc, or both. In each case, we reflect at the consecutive $\times-\bullet$ entries and then arrange the arcs to be of $\bullet-\times$ type 
as follows:

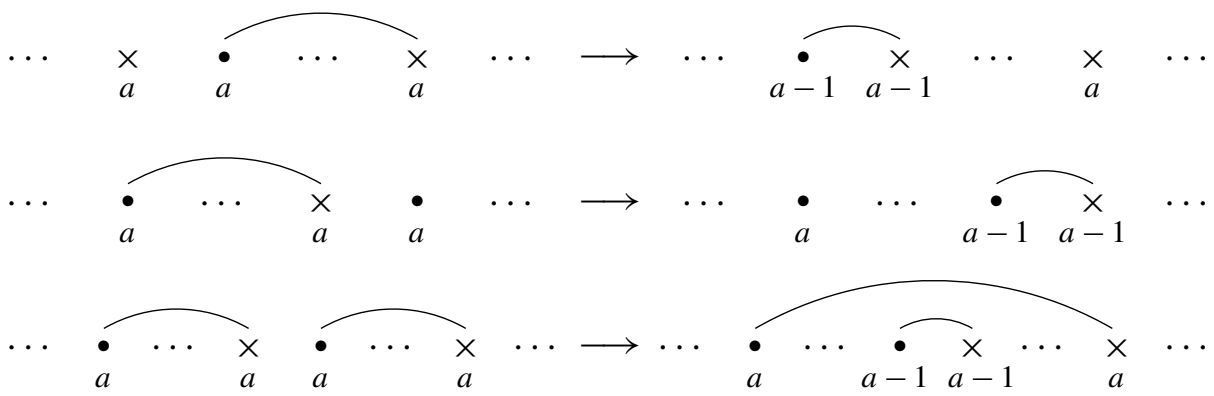

In each case, an atypical entry of $a-1$ was added, but the interval property is preserved since we still have an $a$ in the diagram. The maximality property is also preserved since the new diagram has the same number of arcs as the old diagram. Moreover, the arcs of the new diagram are again nonintersecting and of $\bullet-\times$ type. We proceed to the next $\times$, and continue until each $\times$ is to the right of every $\bullet$.

To prove the other direction, we introduce an algorithm to move from a totally connected weight in the standard choice of simple roots to an admissible choice of simple roots, which we call the shortening algorithm. Let's first illustrate our algorithm with an example.

Example 22. Let us take the following totally connected weight, and show a sequence of odd reflections that brings it to an admissible choice of simple roots:

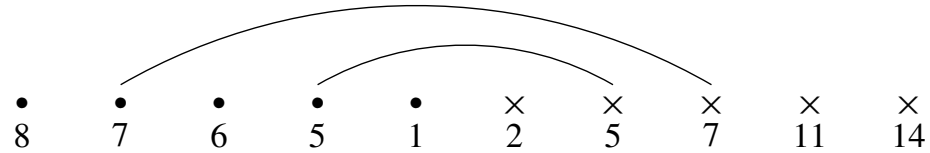

Since the innermost entries 1 and 2 are different from all the other entries, using the odd reflection defined in (2-3) we can move them outside the arcs to the right and left, respectively:

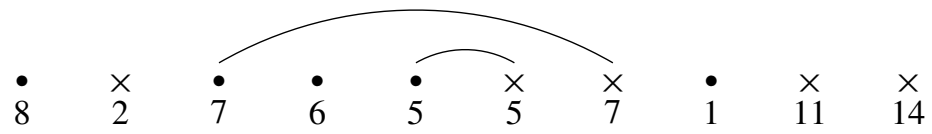

Next, we apply the odd reflection $s_{\varepsilon_{4}-\delta_{2}}$, and then choose the arc arrangement to be of $\bullet-\times$ type so that we can move the extra 6 outside of the arcs:

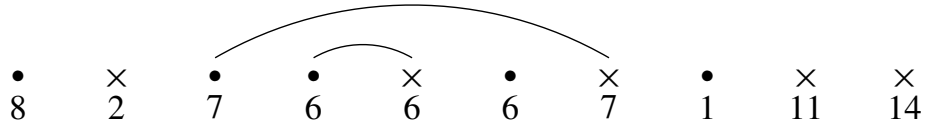


Then we move the $6 \bullet$-entry to the right outside of the arcs:

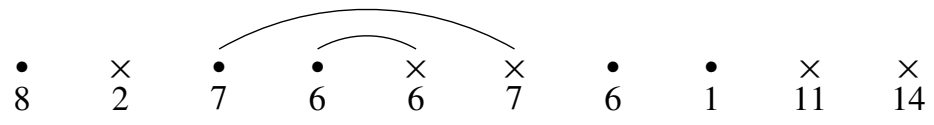

Finally, we apply $s_{\varepsilon_{3}-\delta_{2}}$, and then arrange the arcs to be short, obtaining an admissible choice of simple roots:

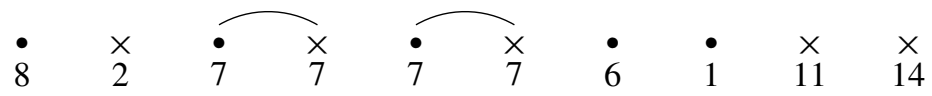

Proof of Theorem 21 " $\Leftarrow$ ". We give an algorithm to move from a totally connected weight $\lambda_{\text {st }}$ of a finite-dimensional simple module to an admissible choice of simple roots using a sequence of odd reflections. The main idea is to push all the typical entries which are below the arcs to the side, making all the atypical entries the same. This will allow us to choose an arc arrangement with only short arcs.

We have from Remark 10 that adjacent $\bullet$-entries of $\lambda_{\pi}^{\rho}$ are strictly decreasing, while adjacent $\times$-entries of $\lambda_{\pi}^{\rho}$ are strictly increasing. Hence, in the standard arc diagram all equalities between entries correspond to arcs, and the arcs are nested in each other. Moreover, all of the entries below the innermost arc are typical and are different from the rest of the entries. Before applying the algorithm, we move these entries outside of the arcs by pushing the $\times$ 's to the left and the $\bullet$ 's to the right, which makes the innermost arc short and of $\bullet-\times$ type.

We begin the algorithm by reflecting along the innermost arc and then we arrange the arcs to be of $\bullet-\times$ type. Due to the interval property there are three possibilities: either there is an $a+1 \bullet$-entry on the left, an $a+1 \times$-entry on the right, or both, in which case they must be connected by an arc:

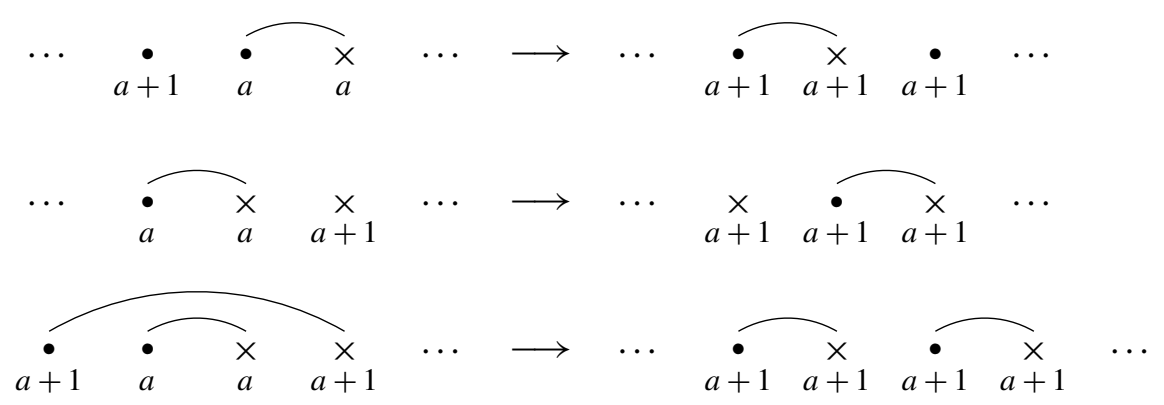

After the reflection, in the first case we push the unmatched $a+1 \bullet$-entry to the right outside of the arcs, and in the second case we push the unmatched $a+1$ $x$-entry to the left outside of the arcs.

This will be the base of our induction. Suppose that after $k$ steps all the atypical entries below $a+k+1$ are now equal to $a+k$ and are paired by short arcs, and 
all other entries which are below an arc remained as in the original diagram of $\lambda_{\text {st }}^{\rho}$. Due to the interval property there are now three possibilities, namely, either there is an $a+k+1 \bullet$-entry on the left, or an $a+k+1 \times$-entry on the right, or both, in which case they must be connected by an arc:
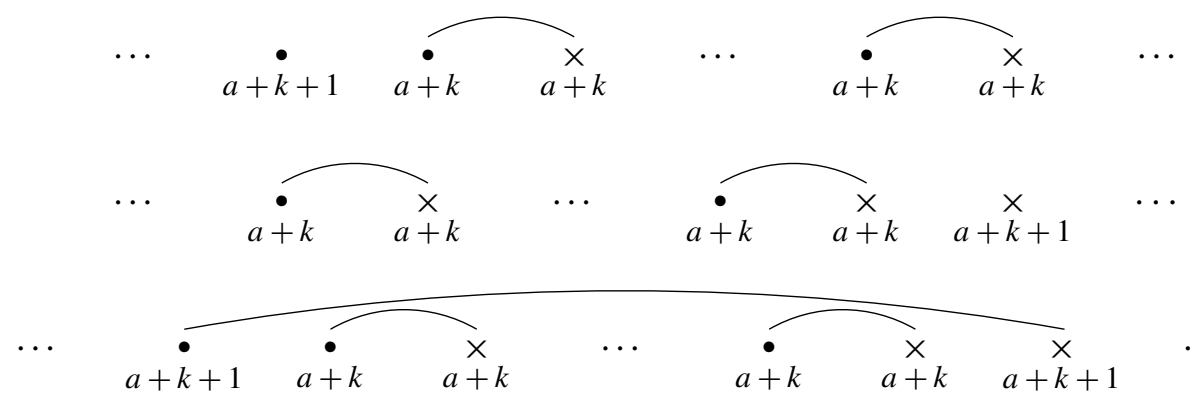

We reflect along the short arcs and arrange the arcs to get the following three diagrams, respectively:

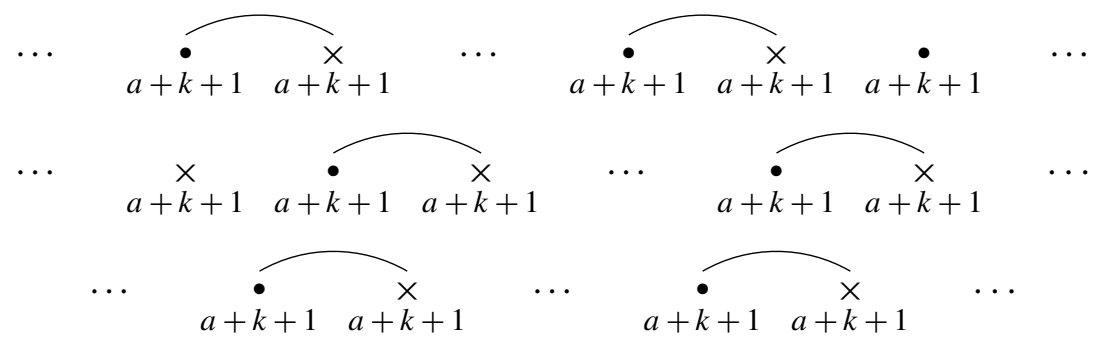

In the first case we push the unmatched $a+k+1 \bullet$-entry to the right outside of the arcs, and in the second case we push the unmatched $a+k+1 \times$-entry to the left outside of the arcs.

We continue this procedure until we reach the outermost arc. After doing the last step, all of the arcs become short and we get an admissible choice of simple roots. Moreover, all the atypical entries are now adjacent and equal to the largest atypical entry of $\lambda_{\mathrm{st}}^{\rho}$. The typical $\bullet$-entries (resp. $\times$-entries) which were under an $\operatorname{arc}$ of $\lambda_{\text {st }}^{\rho}$ were pushed to the right (resp. left).

Remark 23. For each $\lambda_{\text {st }}^{\rho}$ which corresponds to a totally connected $\lambda$ in $\pi_{\text {st }}$, one can immediately determine the arc diagram given by shortening algorithm. For example, if $\lambda_{\mathrm{st}}^{\rho}$ corresponds to the arc diagram

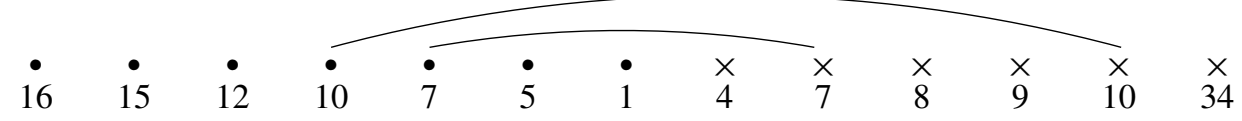


then the shortening algorithm gives

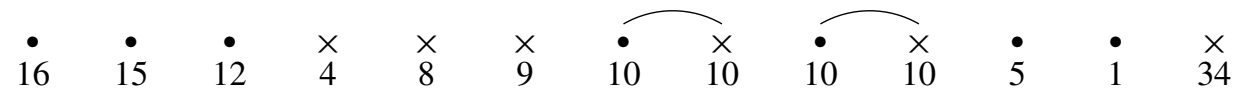

where the typical $\bullet$-entries under an arc of $\lambda_{\mathrm{st}}^{\rho}$ are pushed to the right outside the arcs, the typical $\times$-entries under an arc of $\lambda_{\mathrm{st}}^{\rho}$ are pushed to left outside the arcs, the atypical entries are set equal to the maximal atypical entry of $\lambda_{\mathrm{st}}^{\rho}$, and then all arcs are chosen to be short.

Definition 24. We call an arc diagram for $L$ the special arc diagram if:

(1) All arcs are short, of $\bullet-\times$ type and are adjacent.

(2) All atypical entries are equal.

(3) The typical nodes at each end of the diagram are organized so that the •'s precede the $\times$ 's.

(4) The -entries are strictly decreasing left to right, except for atypical entries which are all equal.

(5) The $\times$-entries are strictly increasing left to right, except for atypical entries which are all equal.

Remark 25. The arc diagram obtained in the last step of the shortening algorithm is a special arc diagram for $L$, since it satisfies (1)-(5). Hence, every KW-module has a special set of simple roots, since the highest weight of a KW-module with respect to $\pi_{\mathrm{st}}$ is totally connected. Moreover, it is unique since we can apply the reverse of the shortening algorithm to a special arc diagram to obtain the standard arc diagram for a totally connected weight $\lambda$ of a finite-dimensional module.

\section{The Su-Zhang character formula for the totally connected case}

We use Brundan's algorithm to characterize KW-modules in terms of KazhdanLusztig polynomials and to prove the Su-Zhang character formula for finitedimensional simple modules with a totally connected highest weight in the standard choice of simple roots $\pi_{\mathrm{st}}$. Recall the notation $\left(\lambda_{\mathrm{st}}^{\rho}\right)^{\Uparrow}$ and $|\nu|_{S_{\lambda}}$ from Section 2.3.

Theorem 26 [Su and Zhang 2007, 4.13]. Let $\lambda_{\text {st }}$ be a totally connected weight with $a \lambda^{\rho}$-maximal isotropic set $S_{\lambda}$ such that $\left|S_{\lambda}\right|=r$. Then

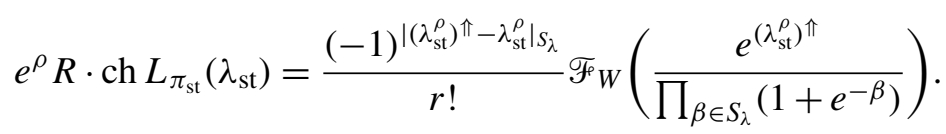

To prove this theorem, we extend the ring $\mathscr{E}$ by adding expansions of the elements $1 /\left(1+e^{-\beta}\right)$ for $\beta \in \Delta_{\overline{1}}^{+}$with respect to $\pi_{\mathrm{st}}$ as geometric series in the domain $\left|e^{-\beta}\right|<1$. Since $\Delta_{\overline{1}}^{+}$is fixed by $W$, expanding commutes with the action of $W$. 
4.1. Brundan's algorithm. Serganova [1996] introduced the generalized KazhdanLusztig polynomials to give a character formula for finite-dimensional irreducible representations of $\mathfrak{g l}(m \mid n)$. For each $\lambda$ and $\mu$ dominant integral, the KazhdanLusztig polynomial $K_{\lambda, \mu}(q)$ was shown to yield the multiplicity of Kac module $\bar{L}(\mu)$ inside the simple module $L_{\text {st }}(\lambda)$ in the following sense:

$$
\operatorname{ch} L_{\pi_{\mathrm{st}}}(\lambda)=\sum_{\mu \in \mathfrak{h}^{*}} K_{\lambda, \mu}(-1) \operatorname{ch} \bar{L}(\mu)
$$

In this section we recall the algorithm of [Brundan 2003] computing $K_{\lambda, \mu}(q)$ in terms of weight diagrams.

We define a right move map from the set of (labeled) weight diagrams to itself in two steps: Let $D_{\mu}$ be a weight diagram for $\mu \in \mathbb{P}^{+}$, and choose a labeling of the $\times$ 's with indexing set $\{1, \ldots, r\}$. Then, for each $\times$, starting with the rightmost $\times$, "mark" the next empty spot to the right of it (which is unmarked). The right move $R_{i}$ is then defined by moving $\times_{i}$ to the empty spot it marked.

Example 27. Let $D_{\mu}$ be

$$
\begin{array}{lllllllllllllllll}
\ldots & -1 & 0 & 1 & 2 & 3 & \times & 3 & \times & 6 & 7 & 8 & 9 & 10 & 11 & 12 & \ldots
\end{array}
$$

The rightmost $\times$ is at 8 and we mark 11 for it. The next $\times$ is at 6 and so we mark 7 . Finally for the leftmost $\times$ we mark 12 . Then

$$
\begin{array}{llllllllllllllllll}
R_{1}\left(D_{\mu}\right)=\cdots & -1 & 0 & 1 & 2 & 3 & 4 & 5 & 6 & 7 & 8 & 9 & 10 & 11 & 12 & \cdots \\
R_{2}\left(D_{\mu}\right)=\cdots & -1 & 0 & 1 & 2 & 3 & 4 & 5 & 6 & 7 & 8 & 9 & 10 & 11 & 12 & \cdots \\
R_{3}\left(D_{\mu}\right)=\cdots & -1 & 0 & 1 & 2 & 3 & 4 & 5 & 6 & 7 & 8 & 9 & 10 & 11 & 12 & \cdots
\end{array}
$$

Note that the weight $\mu_{i}^{\rho}$ corresponding to $R_{i}\left(D_{\mu}\right)$ does not only differ from $\mu^{\rho}$ by atypical roots. It also has a different atypical set. In the previous example

$$
\begin{aligned}
S_{\mu} & =\left\{\varepsilon_{3}-\delta_{2}, \varepsilon_{4}-\delta_{3}, \varepsilon_{6}-\delta_{4}\right\}, \\
S_{\mu_{1}} & =\left\{\varepsilon_{1}-\delta_{2}, \varepsilon_{4}-\delta_{3}, \varepsilon_{5}-\delta_{4}\right\} .
\end{aligned}
$$

Definition 28. Let $\mu, \lambda \in \mathbb{P}^{+}$, and label the $\times$'s in the diagram $D_{\mu}$ from left to right with $1, \ldots, r$. A right path from $D_{\mu}$ to $D_{\lambda}$ is a sequence of right moves $\theta=R_{i_{1}} \circ \cdots \circ R_{i_{k}}$, where $i_{1} \leq \ldots \leq i_{k}$ and $\theta\left(D_{\mu}\right)=D_{\lambda}$. The length of the path is $l(\theta):=k$.

Example 29. Let $D_{\mu}$ be

$$
\begin{array}{llllllllllllllllll}
\ldots & -1 & 0 & 1 & 2 & 3 & 4 & 5 & 6 & 7 & 8 & 9 & 10 & 11 & 12 & \ldots
\end{array}
$$


and $D_{\lambda}$ be as in Example 27. The boxes in the diagram represent the locations of the $\times$ 's in $\lambda$. Then there are two paths from $D_{\mu}$ to $D_{\lambda}$, namely

$$
\begin{aligned}
& D_{\lambda}=R_{1} \circ R_{1} \circ R_{2} \circ R_{3} \circ R_{3}\left(D_{\mu}\right), \\
& D_{\lambda}=R_{1} \circ R_{1} \circ R_{2}\left(D_{\mu}\right) .
\end{aligned}
$$

The first path sends the $i$-th $\times$ of $\mu$ to the $i$-th box, whereas the second path permutes the order. Not all such permutations are valid, for example the third $\times$ in $\mu$ cannot be moved to the left. Also suppose the second and third $\times$ 's will remain in the first and second boxes, respectively. Then the first $\times$ will never reach the box at 8 since it is marked by the $\times$ at 4 , and hence such a path would be invalid.

If there exist paths from $D_{\mu}$ to $D_{\lambda}$, then one of them sends the $i$-th $\times$ of $\mu^{\rho}$ to the location of the $i$-th $\times$ of $\lambda^{\rho}$. This path is unique because the $\times$ 's are moved in order. We call it the trivial path from $D_{\mu}$ to $D_{\lambda}$ and denote its length by $l_{\lambda, \mu}$ (this was denoted as $l(\lambda, \mu)$ in [Su and Zhang 2007, (3.15)]).

The trivial path is strictly longer than the rest of the paths. Indeed, in other paths, there is at least one $\times$ which is not moved as far as possible. This implies that another $\times$ will jump over it, making the move longer. So in this case one needs less moves to fill all of the boxes.

By [Brundan 2003, Corollary 3.39], we have

$$
K_{\lambda, \mu}(q)=\sum_{\theta \in P} q^{l(\theta)}
$$

where $P$ is the set of paths from $D_{\mu}$ to $D_{\lambda}$. In the previous example $K_{\lambda, \mu}=q^{5}+q^{3}$.

\subsection{Kazhdan-Lusztig polynomials and the Su-Zhang character formula. First} we show that $\lambda$ being totally connected is equivalent to all paths to $D_{\lambda}$ being trivial. This yields a new characterization of KW-modules in terms of the KazhdanLusztig polynomials. Then we use Brundan's algorithm to give a closed formula for $e^{\rho} R \cdot \operatorname{ch} L_{\pi_{\mathrm{st}}}(\lambda)$, which was originally proven in [Su and Zhang 2007, 4.13].

Lemma 30. Let $\lambda \in \mathbb{P}^{+}$. Then $\lambda$ is totally connected if and only if for every $\mu \in \mathbb{P}^{+}$ there is at most one path from $D_{\mu}$ to $D_{\lambda}$.

Proof. We will refer to the locations of the $\times$ 's of the weight diagram $D_{\lambda}$ as boxes, and the paths will send the $\times$ 's in $D_{\mu}$ to boxes.

The weight $\lambda$ is totally connected when there are no empty spots between the boxes. This implies that a path from $D_{\mu}$ to $D_{\lambda}$ must send the $i$-th $\times$ of $\mu$ to the $i$-th box. Indeed, if the $i$-th $\times$ of $\mu$ is sent to the $j$-th box, $j<i$, then the next empty box to the right of the $j$-th box will be marked by the $i$-th $\times$, and so no other $\times$ can be sent there. This implies that the path must be unique. 
Suppose that there exists $\mu$ for which there is a nontrivial path to $D_{\lambda}$. In this path there is an $\times$ of $\mu$, say the $i$-th, which is sent to the $j$-th box where $j<i$. Then the next empty spot after this box cannot have a box in it. So $\lambda$ is not totally connected.

Corollary 31. A module $L_{\pi_{\mathrm{st}}}(\lambda)$ is a $K W$-module if and only if all its KazhdanLusztig polynomials are monomials. In this case, $K_{\lambda, \mu}(q)=q^{l_{\lambda, \mu} \text { : }}$

From (4-2) and (2-5) we also obtain the following, which is a special case of [Su and Zhang 2007, Theorem 4.1].

Corollary 32. Suppose $\lambda$ is totally connected. Since the unique path from $D_{\mu}$ to $D_{\lambda}$ is the trivial one, we get

$$
e^{\rho} R \cdot \operatorname{ch} L_{\pi_{\mathrm{st}}}(\lambda)=\sum_{\mu \in P_{\lambda}}(-1)^{l_{\lambda, \mu} \mathscr{F}_{W}}\left(e^{\mu^{\rho}}\right)
$$

where $P_{\lambda} \subset \mathbb{P}^{+}$is the set of $\mu \in \mathbb{P}^{+}$for which there is a path from $D_{\mu}$ to $D_{\lambda}$.

Remark 33. By Corollary 31, KW-modules are the same as what are called Kostant modules in [Brundan and Stroppel 2012]. Brundan and Stroppel [2012] showed that Kostant modules are parametrized in their notation by the weights in which no two vertices labeled $\vee$ have a vertex labeled $\wedge$ in between. This is equivalent to the combinatorial condition given in Remark 19 of this paper. They also proved that all Kostant modules possess a BGG-type resolution, so (4-4) can be realized as the Euler characteristic of this resolution.

Note that for each $\mu \in P_{\lambda}$ the $W$ orbit of $\mu^{\rho}$ intersects $\left(\lambda^{\rho}-\mathbb{N} S_{\lambda}\right)$. We denote by $\bar{\mu}$ the unique maximal element of this intersection with respect to the standard order on $\mathfrak{h}^{*}$. We define

$$
C_{\lambda, \text { reg }}^{\text {Lexi }}:=\left\{\bar{\mu} \mid \mu \in P_{\lambda}\right\}
$$

Since $P_{\lambda} \subset \mathbb{P}^{+}$, this defines a bijection between the sets $P_{\lambda}$ and $C_{\lambda \text {,reg. }}^{\mathrm{Lexi}}$.

For $\mu \in P_{\lambda}$, we can realize $\bar{\mu}$ more explicitly as follows. If $\varepsilon_{i}$ and $\delta_{j}$ are typical nodes of $\lambda^{\rho}$, then $\left(\bar{\mu}, \varepsilon_{i}\right):=\left(\lambda^{\rho}, \varepsilon_{i}\right)$ and $\left(\bar{\mu}, \delta_{j}\right):=\left(\lambda^{\rho}, \delta_{j}\right)$. The location of the atypical entries for $\bar{\mu}$ is determined by locations in $\lambda^{\rho}$. The set of atypical entries of $\bar{\mu}$ correspond to the set of atypical entries of $\mu^{\rho}$, ordered such that the $\varepsilon$-atypical entries are decreasing and the $\delta$-atypical entries are increasing. In particular,

$C_{\lambda, \text { reg }}^{\text {Lexi }}=\left\{v \in \lambda^{\rho}-\sum_{i=1}^{r} \mathbb{N} \beta_{i} \mid v_{\beta_{1}}<v_{\beta_{2}}<\cdots<v_{\beta_{r}}\right.$ and $w(v) \in \mathbb{P}^{+}$for some $\left.w \in W\right\}$. 
Example 34. Consider

$$
\begin{aligned}
\lambda^{\rho} & =10 \varepsilon_{1}+9 \varepsilon_{2}+\underline{8 \varepsilon_{3}}+\underline{6 \varepsilon_{4}}+5 \varepsilon_{5}+\underline{4 \varepsilon_{6}}-2 \delta_{1}-\underline{4 \delta_{2}}-\underline{6 \delta_{3}}-\underline{8 \delta_{4}}, \\
\mu^{\rho} & =10 \varepsilon_{1}+9 \varepsilon_{2}+\underline{6 \varepsilon_{3}}+5 \varepsilon_{4}+\underline{4 \varepsilon_{5}}+\underline{\varepsilon_{6}}-\underline{\delta_{1}}-2 \delta_{2}-\underline{4 \delta_{3}}-\underline{6 \delta_{4}}, \\
\bar{\mu} & =10 \varepsilon_{1}+9 \varepsilon_{2}+\underline{6 \varepsilon_{3}}+\underline{4 \varepsilon_{4}}+5 \varepsilon_{5}+\underline{\varepsilon_{6}}-2 \delta_{1}-\underline{\delta_{2}}-\underline{4 \delta_{3}}-\underline{6 \delta_{4}} .
\end{aligned}
$$

Here $S_{\lambda}=\left\{\varepsilon_{6}-\delta_{2}, \varepsilon_{4}-\delta_{3}, \varepsilon_{3}-\delta_{4}\right\}$ and $\lambda^{\rho}=\bar{\mu}+3\left(\varepsilon_{6}-\delta_{2}\right)+2\left(\varepsilon_{4}-\delta_{3}\right)+2\left(\varepsilon_{3}-\delta_{4}\right)$.

Remark 35. The element $w \in W$ for which $w\left(\mu^{\rho}\right)=\bar{\mu}$ can be described explicitly in terms of the trivial path $\theta$. Define $\theta=R_{i_{1}} \circ \cdots \circ R_{i_{N}}$; then $w=w_{1} \cdots \cdots w_{N}$, where each $w_{j}$ is defined as follows: Suppose that the move $R_{i_{j}}$ moved the $\times$ at $n_{j}$ to an empty spot at $n_{j}+k_{j}$, namely, it skipped over $k_{j}-1$ spots with >'s and $<$ 's. Then $w_{j}=s_{1} \cdots s_{k_{j}-1}$, where $s_{i}$ is of the form $s_{\varepsilon_{l}-\varepsilon_{l+1}}$ if the $i$-th skip is over the $>$ of $\varepsilon_{l}$ and is of the form $s_{\delta_{l}-\delta_{l+1}}$ if it is over the $<$ of $\delta_{l}$. In particular, $l\left(w_{j}\right)=k_{j}-1$. Moreover $l(w)=\sum l\left(w_{i}\right)$.

The following lemma is the main step of the proof, in which we move from an algorithmic formula to a closed one, and it is a special case of [Su and Zhang 2007, Theorem 4.2].

Lemma 36.

$$
e^{\rho} R \cdot \operatorname{ch} L_{\pi_{\mathrm{st}}}(\lambda)=\sum_{\bar{\mu} \in C_{\lambda, \mathrm{reg}}^{\text {Lexi }}}(-1)^{\left|\lambda^{\rho}-\bar{\mu}\right| S_{\lambda} \mathscr{F}_{W}}\left(e^{\bar{\mu}}\right) .
$$

Proof. Let us show that for each $\mu \in P_{\lambda}$

$$
(-1)^{l_{\lambda, \mu} \mathscr{F}_{W}}\left(e^{\mu^{\rho}}\right)=(-1)^{\left|\lambda^{\rho}-\bar{\mu}\right|_{\lambda} \mathscr{F}_{W}}\left(e^{\bar{\mu}}\right) .
$$

Let $w \in W$ such that $w\left(\mu^{\rho}\right)=\bar{\mu}$. We claim that $\left|\lambda^{\rho}-\bar{\mu}\right|_{S_{\lambda}}=l_{\lambda, \mu}+l(w)$, which proves (4-5). Indeed, the number $\left|\lambda^{\rho}-\bar{\mu}\right|_{S_{\lambda}}$ is the sum of the differences between the atypical entries of $\lambda^{\rho}$ and $\bar{\mu}$. This is equal to the number of moves in the trivial path $l_{\lambda, \mu}$ plus the total number of spots being skipped. By Remark 35, $l(w)$ is exactly the number of spots skipped in the trivial path.

Proof of Theorem 26. Our proof goes as follows. First, we enlarge the set $C_{\lambda, \text { reg }}^{\text {Lexi by }}$ adding elements which are annihilated by $\mathscr{F}_{W}$; we call this new set $C_{\lambda}^{\text {Lexi }}$. Then we express $C_{\lambda}^{\text {Lexi }}$ in terms of $\left(\lambda_{\mathrm{st}}^{\rho}\right) \Uparrow$. Finally, we add more summands to the expression

$$
\sum_{\bar{\mu} \in C_{\lambda}^{\text {Lexi }}}(-1)^{\left|\lambda^{\rho}-\bar{\mu}\right|_{\lambda} \mathscr{F}_{W}}\left(e^{\bar{\mu}}\right)
$$

which are annihilated by $\mathscr{F}_{W}$ but that allow us to write the sum in a nicer way.

Denote the $\lambda^{\rho}$-maximal atypical set by $S_{\lambda}=\left\{\beta_{1}, \ldots, \beta_{r}\right\}$, where the elements $\beta_{i}=\varepsilon_{s_{i}}-\delta_{t_{i}}$ are ordered such that $t_{i}<t_{i+1}$. Let

$$
C_{\lambda}^{\text {Lexi }}=\left\{v \in \lambda^{\rho}-\sum_{i=1}^{r} \mathbb{N} \beta_{i} \mid v_{\beta_{1}}<v_{\beta_{2}}<\cdots<v_{\beta_{r}}\right\} .
$$


Then $C_{\lambda}^{\text {Lexi }}$ can be expressed as follows. Let $k_{1}, \ldots, k_{r} \in \mathbb{N}$ be such that $\lambda^{\rho}=$ $\left(\lambda^{\rho}\right)^{\Uparrow}-\sum_{i=1}^{r} k_{i} \beta_{i}$. Then $k_{1}>\cdots>k_{r}=0$. Let $a_{1}<\cdots<a_{r}$ be the atypical entries of $\lambda^{\rho}$. Then $a_{i}+k_{i}=a_{j}+k_{j}$, and we have

$$
\begin{aligned}
C_{\lambda}^{\text {Lexi }} & =\left\{\lambda^{\rho}-\sum_{i=1}^{r} n_{i} \beta_{i} \mid n_{i} \in \mathbb{N}, a_{1}-n_{1}<a_{2}-n_{2}<\cdots<a_{r}-n_{r}\right\} \\
& =\left\{\lambda^{\rho}-\sum_{i=1}^{r} n_{i} \beta_{i} \mid n_{i} \in \mathbb{N}, k_{1}+n_{1}>k_{2}+n_{2}>\cdots>k_{r}+n_{r}\right\} \\
& =\left\{\left(\lambda^{\rho}\right)^{\Uparrow}-\sum_{i=1}^{r}\left(k_{i}+n_{i}\right) \beta_{i} \mid n_{i} \in \mathbb{N}, k_{1}+n_{1}>k_{2}+n_{2}>\cdots>k_{r}+n_{r}\right\} \\
& =\left\{\left(\lambda^{\rho}\right)^{\Uparrow}-\sum_{i=1}^{r} m_{i} \beta_{i} \mid m_{i} \in \mathbb{Z}_{\geq k_{i}}, m_{1}>m_{2}>\cdots>m_{r}\right\} .
\end{aligned}
$$

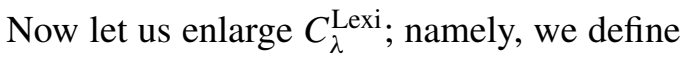

$$
\overline{C_{\lambda}^{\mathrm{Lexi}}}=\left\{\left(\lambda^{\rho}\right)^{\Uparrow}-\sum_{i=1}^{r} m_{i} \beta_{i} \mid m_{i} \in \mathbb{N}, m_{1}>m_{2}>\cdots>m_{r}\right\} .
$$

We claim that

$$
\sum_{\bar{\mu} \in C_{\lambda, \text { reg }}^{\text {Lexi }}}(-1)^{\left|\lambda^{\rho}-\bar{\mu}\right| s_{\lambda} \mathscr{F}_{W}}\left(e^{\bar{\mu}}\right)=\sum_{\nu \in \overline{C_{\lambda}^{\text {Lexi }}}}(-1)^{\left|\lambda^{\rho}-\nu\right| s_{\lambda} \mathscr{F}_{W}}\left(e^{\nu}\right) .
$$

Indeed, if $v \in C_{\lambda}^{\text {Lexi }} \backslash C_{\lambda, \text { reg }}^{\text {Lexi }}$ then the $\varepsilon$ or $\delta$ entries of $v$ are not distinct, so $\mathscr{F}_{W}\left(e^{\nu}\right)=0$. Thus, we are left to show that $\mathscr{F}_{W}\left(e^{\nu}\right)=0$ if $\nu$ is of the form $v=\left(\lambda^{\rho}\right)^{\Uparrow}-\sum_{i=1}^{r} m_{i} \beta_{i} \in$ $\overline{C_{\lambda}^{\text {Lexi }}}$ and $m_{i}<k_{i}$ for some $1 \leq i \leq r$. Indeed, let $j$ be such that $m_{j}<k_{j}$ and $m_{i} \geq k_{i}$ for all $i>j$. Note that since $\lambda$ is totally connected, all the integers between $a_{r}$ and $a_{j}+1$ are entries of $\lambda^{\rho}$. The typical entries of $v$ are the same as of $\lambda^{\rho}$ and there are $r-j+1$ atypical entries which are strictly greater than $a_{j}$. This implies that there must be equal entries of the same type, that is, $v$ has a stabilizer in $W$. Hence, by Lemma 13 and Lemma 2 we conclude that $\mathscr{F}_{W}\left(e^{\nu}\right)=0$.

Let $W_{r}$ be the subgroup of $W$ that permutes $S_{\lambda}$. This subgroup is generated by elements of the form $s_{\varepsilon_{i}-\varepsilon_{j}} s_{\delta_{i^{\prime}}-\delta_{j^{\prime}}}$, where $\varepsilon_{i}-\delta_{i^{\prime}}, \varepsilon_{j}-\delta_{j^{\prime}} \in S_{\lambda}$, so $\left|W_{r}\right|=r$ ! and all $w \in W_{r}$ have positive sign. Hence

$$
\mathscr{F}_{W}\left(\sum_{w \in W_{r}} w e^{v}\right)=r ! \mathscr{F}_{W}\left(e^{v}\right)
$$


for any $v \in \mathfrak{h}^{*}$. Let $W_{r}\left(\overline{C_{\lambda}^{\text {Lexi }}}\right)=\left\{w(v) \mid w \in W_{r}, v \in \overline{C_{\lambda}^{\text {Lexi }}}\right\}$. Then

$$
W_{r}\left(\overline{C_{\lambda}^{\mathrm{Lexi}}}\right)=\left\{\left(\lambda^{\rho}\right)^{\Uparrow}-\sum_{i=1}^{r} m_{i} \beta_{i} \mid m_{i} \in \mathbb{N}, m_{i} \neq m_{j} \text { for } i \neq j\right\},
$$

and so elements from $\left(\left(\lambda^{\rho}\right)^{\Uparrow}-\mathbb{N} S_{\lambda}\right) \backslash W_{r}\left(\overline{C_{\lambda}^{\text {Lexi }}}\right)$ have a stabilizer in $W$. Thus,

$$
\begin{aligned}
r !(-1)^{\left|\left(\lambda^{\rho}\right)^{\Uparrow}-\lambda^{\rho}\right| S_{\lambda}} \sum_{\nu \in \overline{C_{\lambda}^{\text {Lexi }}}}(-1)^{\left|\lambda^{\rho}-\nu\right| s_{\lambda}} \mathscr{F}_{W}\left(e^{\nu}\right) & \\
& =\sum_{\nu \in \overline{C_{\lambda}^{\operatorname{Lexi}}}}(-1)^{\left|\left(\lambda^{\rho}\right)^{\Uparrow}-\nu\right| S_{\lambda} \mathscr{F}_{W}}\left(\sum_{w \in W_{r}} e^{w(\nu)}\right) \\
& =\sum_{\nu \in\left(\lambda^{\rho}\right)^{\Uparrow}-\mathbb{N}_{\lambda}}(-1)^{\left|\left(\lambda^{\rho}\right)^{\Uparrow}-\nu\right| S_{\lambda} \mathscr{F}_{W}}\left(e^{\nu}\right) \\
& =\mathscr{F}_{W}\left(\sum_{\nu \in\left(\lambda^{\rho}\right)^{\Uparrow}-\mathbb{N}_{\lambda}}(-1)^{\left|\left(\lambda^{\rho}\right)^{\Uparrow}-v\right| s_{\lambda}} e^{\nu}\right) \\
& =\mathscr{F}_{W}\left(\frac{e^{\left(\lambda^{\rho}\right)^{\Uparrow}}}{\prod_{\beta \in S_{\lambda}}\left(1+e^{-\beta}\right)}\right) .
\end{aligned}
$$

The character formula in the following theorem is motivated by the denominator identity given in [Gorelik et al. 2012, (1.10)] for $\pi_{\mathrm{st}}$, and can be proven using Lemma 36, formula (4-6) and the methods above.

Theorem 37. Let $\lambda_{\text {st }}$ be a totally connected weight with a $\lambda^{\rho}$-maximal isotropic set $\beta_{1}, \ldots, \beta_{r}$ ordered such that $\beta_{i}<\beta_{i+1}$ for $i=1, \ldots, r-1$. Then

$$
e^{\rho} R \cdot \operatorname{ch} L_{\pi_{\mathrm{st}}}(\lambda)=\mathscr{F}_{W}\left(\frac{e^{\left(\lambda^{\rho}\right)^{\Uparrow}}}{\left(1+e^{-\beta_{1}}\right)\left(1-e^{-\beta_{1}-\beta_{2}}\right) \cdots\left(1-(-1)^{r} e^{-\sum_{i=1}^{r} \beta_{i}}\right)}\right) .
$$

\section{Kac-Wakimoto character formula for $\mathrm{KW}$-modules}

5.1. The special case. Let us show that Theorem 26 generalizes to other sets of simple roots by proving that the character formula is preserved under the steps of the shortening algorithm given in the proof of Theorem 21 . This will prove the Kac-Wakimoto character formula for the special set of simple roots.

For a totally connected highest weight $\lambda$ of a finite-dimensional simple module $L_{\pi_{\mathrm{st}}}(\lambda)$, we let $\pi_{k}$ denote the set of simple roots obtained after $k$ steps of the shortening algorithm applied to $\lambda^{\rho}$. Let $\lambda_{\pi_{k}} \in \mathfrak{h}^{*}$ be such that $L=L_{\pi_{k}}\left(\lambda_{\pi_{k}}\right)$. Set $S_{0}=S_{\lambda}$ and let $S_{k}$ be the $\lambda_{\pi_{k}}^{\rho}$-maximal isotropic set corresponding to the arc arrangement obtained by the $k$-th step of the algorithm. Then we have the following: 
Theorem 38. Let $\lambda$ be a totally connected weight and let $L=L_{\pi_{\mathrm{st}}}(\lambda)$. Then

$$
e^{\rho} R \cdot \operatorname{ch} L=\frac{(-1)^{\left|\left(\lambda_{\pi_{k}}^{\rho}\right) \Uparrow-\lambda_{\pi_{k}}^{\rho}\right| s_{k}}}{r !} \mathscr{F}_{W}\left(\frac{e^{\left(\lambda_{\pi_{k}}^{\rho}\right)^{\Uparrow}}}{\prod_{\beta \in S_{k}}\left(1+e^{-\beta}\right)}\right) .
$$

Our proof is by induction on the steps of the shortening algorithm. Let us first see an example:

Example 39. Given $\lambda_{\text {st }}^{\rho}$ corresponding to diagram (3-3) from Example 22, we show that formula (5-1) holds after one step of the shortening algorithm.

To obtain $\left(\lambda_{\mathrm{st}}^{\rho}\right)^{\Uparrow}=\lambda_{\mathrm{st}}^{\rho}+2\left(\varepsilon_{4}-\delta_{2}\right)$ from the $\lambda_{\mathrm{st}}^{\rho}$ diagram, each entry labeled with a 5 should be replaced by a 7 . To start the algorithm, we first push the entries 1 and 2 outside of the arcs, which does not change $\lambda_{\text {st }}^{\rho}$ and $S_{\lambda_{\text {st }}}$ so the formula is clearly preserved.

Next we apply $r_{\varepsilon_{4}-\delta_{2}}$ and obtain $\lambda_{\pi_{1}}^{\rho}$ corresponding to diagram (3-4). Then $\lambda_{\pi_{1}}^{\rho}=\lambda_{\mathrm{st}}^{\rho}+\left(\varepsilon_{4}-\delta_{2}\right)$ and $S_{1}=s_{\varepsilon_{3}-\varepsilon_{4}} S_{\lambda_{\mathrm{st}}}$. So $\left(\lambda_{\pi_{1}}^{\rho}\right) \Uparrow=\lambda_{\pi_{1}}^{\rho}+\left(\varepsilon_{3}-\delta_{2}\right)$ and $\left(\lambda_{\pi_{1}}^{\rho}\right)^{\Uparrow}=$ $s_{\varepsilon_{3}-\varepsilon_{4}}\left(\lambda_{\text {st }}^{\rho}\right)^{\Uparrow}$. Hence

$$
\begin{aligned}
e^{\rho} R \cdot \operatorname{ch} L & =\frac{(-1)^{2}}{2} \mathscr{F}_{W}\left((-1) \cdot s_{\varepsilon_{3}-\varepsilon_{4}} \frac{e^{\left(\lambda_{\mathrm{st}}^{\rho}\right) \Uparrow}}{\prod_{\beta \in S_{\lambda_{\mathrm{st}}}}\left(1+e^{-\beta}\right)}\right) \\
& =\frac{(-1)^{\left|\left(\lambda_{\pi_{1}}^{\rho}\right) \Uparrow-\lambda_{\pi_{1}}^{\rho}\right| S_{1}}}{2} \mathscr{F}_{W}\left(\frac{e^{\left(\lambda_{\pi_{1}}^{\rho}\right)^{\Uparrow}}}{\prod_{\beta \in S_{1}}\left(1+e^{-\beta}\right)}\right),
\end{aligned}
$$

and the formula is preserved. Finally, we move the 6 out to obtain diagram (3-5). Since this does not change $\lambda_{\pi_{1}}^{\rho}$ and $S_{\pi_{1}}$ the formula is preserved.

Proof of Theorem 38. Our proof is by induction on the steps of the shortening algorithm from the proof of Theorem 21. After $k$ steps, we obtain new data $\pi_{k} \lambda_{\pi_{k}}^{\rho}$, $\lambda_{\pi_{k}}, S_{k}$. We express the right-hand side of formula (5-1) in terms of this new data, and prove that it is equal to $e^{\rho} R \cdot \operatorname{ch} L$ using the formula obtained after $k-1$ steps.

Before applying the algorithm we start by pushing the entries located below the innermost arc outside of the arcs. Since this corresponds to reflections with respect to roots which are not orthogonal to $\lambda_{\pi_{\mathrm{st}}}^{\rho}$, this changes $\pi_{\mathrm{st}}$ but not $\lambda_{\pi_{\mathrm{st}}}^{\rho}$ or $S_{\lambda_{\mathrm{st}}}$. So formula (5-1) is unchanged.

Now suppose that the formula holds after $k-1$ steps of the algorithm, that is,

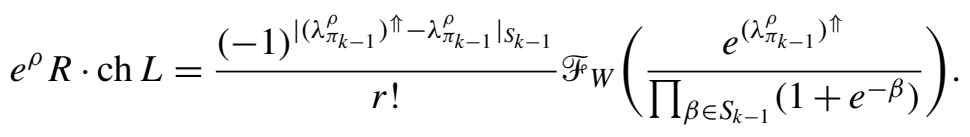

Let us apply one more step and show that (5-2) implies (5-1). There are three cases, depending on the location of the $b+1$ entry (see the proof of the " $\Leftarrow$ " direction of Theorem 21). In each case, we reflect at all of the short arcs, and then arrange the 
arcs to be of $\bullet-\times$ type:

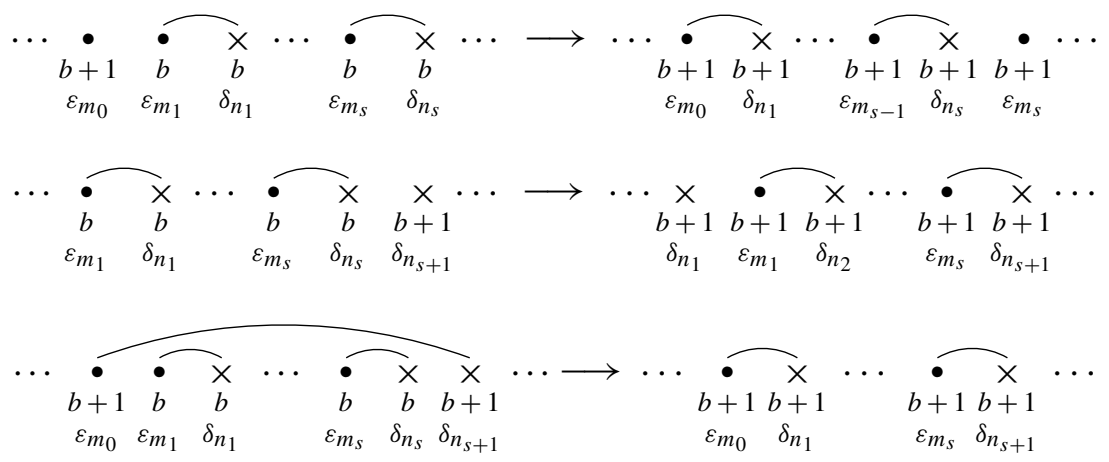

This does not change the typical entries or the maximal atypical entry, so $\left(\lambda_{\pi_{k-1}}^{\rho}\right)^{\Uparrow}$ only differs $\left(\lambda_{\pi_{k}}^{\rho}\right) \Uparrow$ by a permutation of the entries. Moreover, $\lambda_{\pi_{k}}^{\rho}$ is "closer" to $\left(\lambda_{\mathrm{st}}^{\rho}\right) \Uparrow$ than in the previous step because some entries were increased. Indeed, the sequence of odd reflections is with respect to the simple atypical odd roots $S_{k-1} \cap \pi_{k-1}$, so we have

$$
\lambda_{\pi_{k}}^{\rho}=\lambda_{\pi_{k-1}}^{\rho}+\sum_{\beta \in S_{k-1} \cap \pi_{k-1}} \beta .
$$

For each diagram from (5-3), there exists $w \in W$ such that

$$
w S_{k-1}=S_{k}, \quad w\left(\lambda_{\pi_{k-1}}^{\rho}\right)^{\Uparrow}=\left(\lambda_{\pi_{k}}^{\rho}\right)^{\Uparrow}, \quad \text { and } \quad l(w)=\left|S_{k-1} \cap \pi_{k-1}\right| .
$$

Indeed, we have that $S_{k-1} \cap \pi_{k-1}=\left\{\beta_{1}, \ldots, \beta_{s}\right\}$, where each $\beta_{i}=\varepsilon_{m_{i}}-\delta_{n_{i}}$ is a simple atypical root corresponding to a diagram of (5-3). For the first and third case, we take $w \in \operatorname{Sym}_{m}$ such that $w\left(\varepsilon_{m_{i}}\right)=\varepsilon_{m_{i-1}}, w\left(\varepsilon_{m_{0}}\right)=\varepsilon_{m_{s}}$ and all other elements are fixed, and, in the second case, we take $w \in \operatorname{Sym}_{n}$ such that $w\left(\delta_{i}\right)=\delta_{i+1}$, $w\left(\delta_{s+1}\right)=\delta_{1}$ and all other elements are fixed. Then

$$
\begin{aligned}
e^{\rho} R \cdot \operatorname{ch} L & =\frac{(-1)^{\left|\left(\lambda_{\pi_{k-1}}^{\rho}\right)^{\Uparrow}-\lambda_{\pi_{k-1}}^{\rho}\right| S_{k-1}}}{r !} \mathscr{F}_{W}\left(\frac{e^{\left(\lambda_{\pi_{k-1}}^{\rho}\right)^{\Uparrow}}}{\prod_{\beta \in S_{k-1}}\left(1+e^{-\beta}\right)}\right) \\
& =\frac{(-1)^{\left|\left(\lambda_{\pi_{k-1}}^{\rho}\right)^{\Uparrow}-\lambda_{\pi_{k-1}}^{\rho}\right| S_{k-1}+\left|S_{\lambda_{k-1}} \cap \pi_{k-1}\right|} \mathscr{F}_{W}\left((-1)^{l(w)} w \frac{e^{\left(\lambda_{\pi_{k-1}}^{\rho}\right)^{\Uparrow}}}{\prod_{\beta \in S_{k-1}}\left(1+e^{-\beta}\right)}\right)}{r !} \\
& =\frac{(-1)^{\left|\left(\lambda_{\pi_{k}}^{\rho}\right){ }^{\Uparrow}-\lambda_{\pi_{k}}^{\rho}\right| S_{k}}}{r !} \mathscr{F}_{W}\left(\frac{e^{\left(\lambda_{\pi_{k}}^{\rho}\right)^{\Uparrow}}}{\prod_{\beta \in S_{k}}\left(1+e^{-\beta}\right)}\right) .
\end{aligned}
$$

Finally, we push the smallest unmatched entry outside of the arcs. Since this corresponds to reflections with respect to roots which are not orthogonal to $\lambda_{\pi_{k-1}}^{\rho}$, this does not change formula (5-1). 
Since for the special set of simple roots all atypical entries are equal, we conclude the following:

Corollary 40. Let $L$ be a $K W$-module with an admissible choice of simple roots $\pi$, a corresponding highest weight $\lambda$ and a $\lambda^{\rho}$-maximal isotropic subset $S_{\lambda} \subset \pi$, and let $r=\left|S_{\lambda}\right|$. If $\pi$ is the special set, then

$$
e^{\rho} R \cdot \operatorname{ch} L_{\pi}(\lambda)=\frac{1}{r !} \mathscr{F}_{W}\left(\frac{e^{\lambda^{\rho}}}{\prod_{\beta \in S_{\lambda}}\left(1+e^{-\beta}\right)}\right) .
$$

5.2. The general case. Let us prove the Kac-Wakimoto character formula for an arbitrary admissible choice of simple roots by showing that we can move from any admissible choice of simple roots to the special set in a way that preserves the formula in (5-4).

We will use the following four moves on arc diagrams.

(a)

(c)

(d)

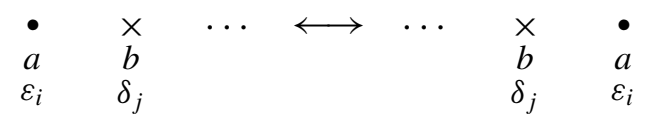

)
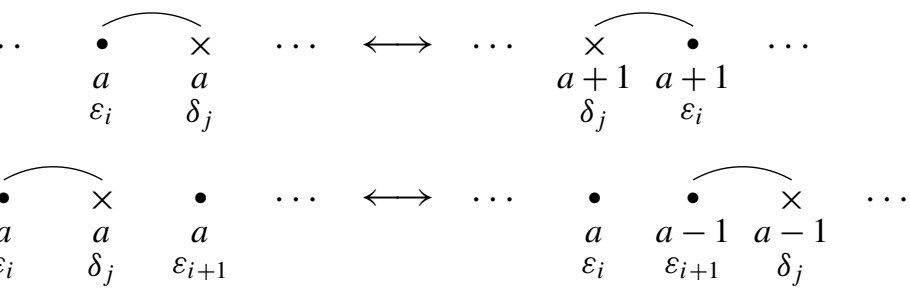

$$
\begin{array}{llllll}
\varepsilon_{i} & \delta_{j} & \varepsilon_{i+1} & \varepsilon_{i} & \varepsilon_{i+1} & \delta_{j}
\end{array}
$$

Each move is achieved by applying an odd reflection to the set of simple roots $\pi \leftrightarrow \pi^{\prime}$ followed by a choice of the arc arrangement. Let us first show that each of these moves preserves the Kac-Wakimoto character formula.

Proposition 41. Suppose that $\left(\pi, \lambda, S_{\lambda}\right)$ and $\left(\pi^{\prime}, \lambda^{\prime}, S_{\lambda^{\prime}}\right)$ differ by one of the above moves. Then

$$
\mathscr{F}_{W}\left(\frac{e^{\lambda^{\rho}}}{\prod_{\beta \in S_{\lambda}}\left(1+e^{-\beta}\right)}\right)=\mathscr{F}_{W}\left(\frac{e^{\lambda^{\rho^{\prime}}}}{\prod_{\beta \in S_{\lambda^{\prime}}}\left(1+e^{-\beta}\right)}\right) .
$$

Proof. We prove the claim for each move defined above. We take $\lambda^{\rho}$ to correspond to the left-hand side diagram and $\lambda^{\rho^{\prime}}$ to correspond to the right-hand side diagram.

The claim is obvious for move (a), since in this case $\lambda^{\rho^{\prime}}=\lambda^{\rho}$ and $S_{\lambda^{\prime}}=S_{\lambda}$.

In move (b), we have $\lambda^{\rho^{\prime}}=\lambda^{\rho}+\left(\varepsilon_{i}-\delta_{j}\right)$, and

$$
S_{\lambda^{\prime}}=\left(S_{\lambda} \backslash\left\{\varepsilon_{i}-\delta_{j}\right\}\right) \cup\left\{\delta_{j}-\varepsilon_{i}\right\},
$$


so

$$
\begin{aligned}
\mathscr{F}_{W}\left(\frac{e^{\lambda^{\rho^{\prime}}}}{\prod_{\beta \in S_{\lambda^{\prime}}}\left(1+e^{-\beta}\right)}\right) & =\mathscr{F}_{W}\left(\frac{e^{\lambda^{\rho}+\varepsilon_{i}-\delta_{j}}}{\prod_{\beta \in S_{\lambda \backslash\left(\varepsilon_{i}-\delta_{j}\right)}}\left(1+e^{-\beta}\right)\left(1+e^{-\left(\delta_{j}-\varepsilon_{i}\right)}\right)}\right) \\
& =\mathscr{F}_{W}\left(\frac{e^{\lambda^{\rho}}}{\prod_{\beta \in S_{\lambda}}\left(1+e^{-\beta}\right)}\right),
\end{aligned}
$$

as desired.

In move (c), we have $\lambda^{\rho^{\prime}}=\lambda^{\rho}+\left(\delta_{j}-\varepsilon_{i+1}\right)$, and

$$
S_{\lambda^{\prime}}=\left(S_{\lambda} \backslash\left\{\varepsilon_{i}-\delta_{j}\right\}\right) \cup\left\{\varepsilon_{i+1}-\delta_{j}\right\},
$$

So

$$
\begin{aligned}
\mathscr{F}_{W}\left(\frac{e^{\lambda^{\rho^{\prime}}}}{\prod_{\beta \in S_{\lambda^{\prime}}}\left(1+e^{-\beta}\right)}\right) & =\mathscr{F}_{W}\left(\frac{e^{\lambda^{\rho}-\varepsilon_{i+1}+\delta_{j}}}{\prod_{\beta \in S_{\lambda \backslash\left(\varepsilon_{i}-\delta_{j}\right)}}\left(1+e^{-\beta}\right)\left(1+e^{-\left(\varepsilon_{i+1}-\delta_{j}\right)}\right)}\right) \\
& =\mathscr{F}_{W}\left(s_{\varepsilon_{i}-\varepsilon_{i+1}}\left(\frac{e^{\lambda^{\rho}-\varepsilon_{i}+\delta_{j}}}{\prod_{\beta \in S_{\lambda}}\left(1+e^{-\beta}\right)}\right)\right) \\
& =-\mathscr{F}_{W}\left(\frac{e^{\lambda^{\rho}-\varepsilon_{i}+\delta_{j}}}{\prod_{\beta \in S_{\lambda}}\left(1+e^{-\beta}\right)}\right) .
\end{aligned}
$$

It remains to show that

$$
-\mathscr{F}_{W}\left(\frac{e^{\lambda^{\rho}-\varepsilon_{i}+\delta_{j}}}{\prod_{\beta \in S_{\lambda}}\left(1+e^{-\beta}\right)}\right)=\mathscr{F}_{W}\left(\frac{e^{\lambda^{\rho}}}{\prod_{\beta \in S_{\lambda}}\left(1+e^{-\beta}\right)}\right),
$$

which we obtain from the following:

$$
\begin{aligned}
\mathscr{F}_{W}\left(\frac{e^{\lambda^{\rho}}}{\prod_{\beta \in S_{\lambda}}\left(1+e^{-\beta}\right)}+\frac{e^{\lambda^{\rho}-\varepsilon_{i}+\delta_{j}}}{\prod_{\beta \in S_{\lambda}}\left(1+e^{-\beta}\right)}\right) & =\mathscr{F}_{W}\left(e^{\lambda^{\rho}} \frac{1+e^{-\left(\varepsilon_{i}-\delta_{j}\right)}}{\prod_{\beta \in S_{\lambda}}\left(1+e^{-\beta}\right)}\right) \\
& =\mathscr{F}_{W}\left(\frac{e^{\lambda^{\rho}}}{\prod_{\beta \in S_{\lambda} \backslash\left\{\varepsilon_{i}-\delta_{j}\right\}}\left(1+e^{-\beta}\right)}\right) \\
& =0 .
\end{aligned}
$$

The last equality holds by Lemma 13 , since the argument of $\mathscr{F}_{W}$ is preserved by the simple reflection $s_{\varepsilon_{i}-\varepsilon_{i-1}}$, which completes the proof for the third move.

In move $(\mathrm{d})$, we have $\lambda^{\rho^{\prime}}=\lambda^{\rho}+\left(\delta_{j-1}-\varepsilon_{i}\right)$, and

$$
S_{\lambda^{\prime}}=\left(S_{\lambda} \backslash\left\{\varepsilon_{i}-\delta_{j}\right\}\right) \cup\left\{\varepsilon_{i}-\delta_{j-1}\right\}
$$

The proof in this case is analogous to the third move, except that the roles of the $\varepsilon$ 's and $\delta$ 's are interchanged. 
Recall the definition of the special set of simple roots given in Definition 24.

Proposition 42. One can move from any admissible choice of simple roots to the special set of simple roots in a way that preserves the character formula (5-4).

First we give an example:

Example 43. Start with the following arc diagram corresponding to an admissible choice of simple roots:

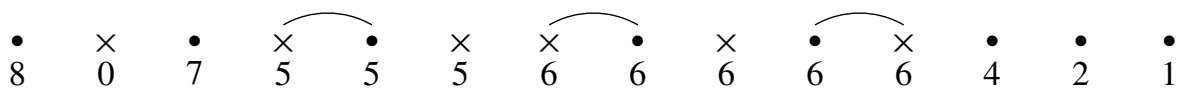

Use move (b) twice to arrange the arcs to be of $\bullet-\times$ type:

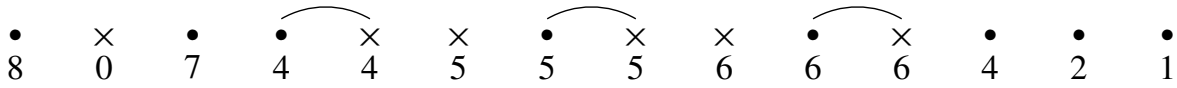

Use move (d) three times to take all the intermediate $\times$ 's outside the arcs:

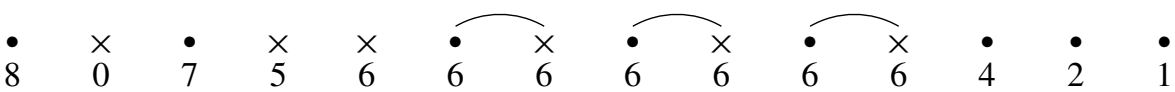

Use move (a) on each side of the arcs to organize the typical entries to have the •'s precede the $\times$ 's:

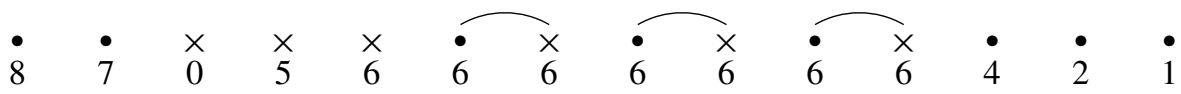

Use moves (d) and (a) to move the typical $\times$-entry 6 to the right of the arcs:

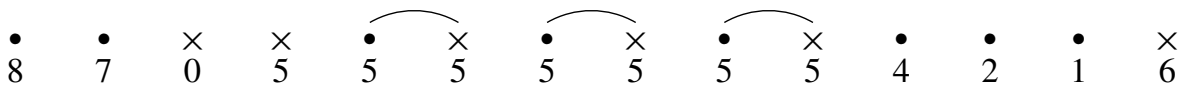

Use moves (d) and (a) to move the typical $\times$-entry 5 to the right of the arcs:

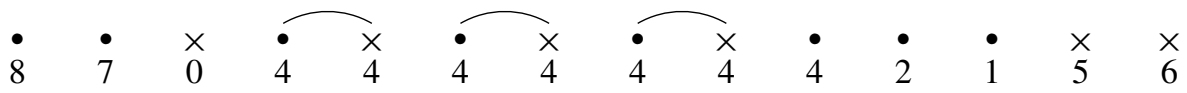

Use moves (c) and (a) to move the typical $\times$-entry 4 to the left of the arcs:

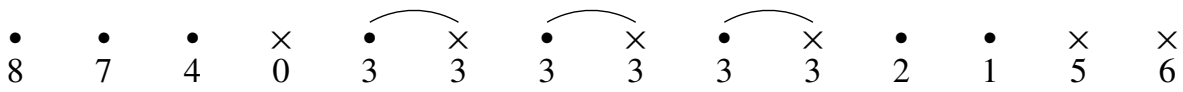

The resulting diagram is the special arc diagram. 
Proof of Proposition 42. We show that we can move from an arbitrary admissible choice of simple roots to the special set in a way that uses only moves (a)-(d) defined above. Since each of these moves was shown to preserve the character formula in Proposition 41, the result then follows.

Consider an arc diagram corresponding to an arbitrary admissible choice of simple roots. Since all arcs are short, we can use move (b) to arrange all of the arcs to be of $\bullet-\times$ type. Then we use move (c) or move (d) to place all the arcs next to each other, which is possible due to Lemma 14. Then, by Lemma 14, all of the atypical entries are equal, and we denote them by $a$. Then on each side of the arcs we use move (a) to organize the typical entries so that the $\bullet$ 's precede the $\times$ 's.

Since the diagram corresponds to a finite-dimensional module, we have at each end that the typical $\times$-entries are strictly increasing, while the typical $\bullet$-entries are strictly decreasing (see Remark 10). Moreover, we can show that, on the left end, the $\times$-entries are $\leqslant a$ while the $\bullet$-entries are $>a$. Indeed, if the biggest $\times$-entry is greater than $a$, then the node can be moved inside the first arc by using move (a), and we reach a contradiction on the value of the adjacent $\times$ entries. Also, if the smallest - -entry is not smaller than $a$, the node can be moved next to the first arc by using move (a), and the same problem arises. Similarly, on the right end the $\times$-entries are $>a$ while the $\bullet$-entries are $\leqslant a$. Together with the fact that no additional arcs are possible by the maximality property, this implies that all typical entries are distinct.

The only way that the resulting diagram is not the special arc diagram is if one of the two inequalities above is an equality, and, in particular, if one of the typical entries equals $a$. If it is the first one, then we use move (d) to transfer the relevant $x$ to the right of the arcs and then move (a) to transfer it to the right of all •'s. If it is the second one, then we use move (c) to transfer the relevant $\bullet$ to the left of the arcs and then move (a) to transfer it to the left of all $\times$ 's. The resulting diagram satisfies the properties of the previous paragraph with the atypical entries now labeled by $a-1$.

We repeat the above step until we cannot do it anymore. After each step the atypical entries are decreased by 1 , while the labeling set for the typical entries remains the same. Hence the process must terminate, and the resulting diagram will be the special arc diagram.

When combined with Corollary 40, this concludes the proof of the Kac-Wakimoto character formula for $\mathfrak{g l}(m \mid n)$ (Theorem 1).

\section{A determinantal character formula for $\mathrm{KW}$-modules of $\mathfrak{g l}(m \mid n)$}

In this section, we use the Kac-Wakimoto character formula to prove a determinantal character formula for $\mathrm{KW}$-modules for $\mathfrak{g l}(m \mid n)$, which is motivated by the 
determinantal character formula proven in [Moens and Van der Jeugt 2004] for critical modules labeled by nonintersecting composite partitions. Our determinantal character formula can be expressed using the data of the special arc diagram for a KW-module $L$ (recall Definition 24).

6.1. A determinantal character formula. Consider the special arc diagram of a KW-module $L$.

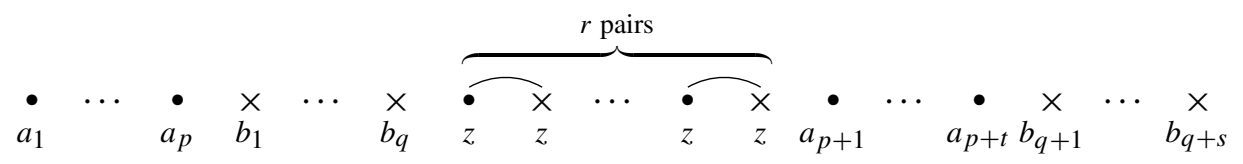

Note that $p+t=m-r$ and $q+s=n-r$. We set $x_{i}=e^{\varepsilon_{i}}$ and $y_{j}=e^{-\delta_{j}}$, and we let

$$
X=\left(x_{i}^{a_{j}}\right)_{\substack{1 \leq i \leq m \\ 1 \leq j \leq m-r}}, \quad Y=\left(y_{j}^{b_{i}}\right)_{\substack{1 \leq i \leq n-r \\ 1 \leq j \leq n}}, \quad Z=\left(\frac{x_{i}^{z} y_{j}^{z}}{1+\left(x_{i} y_{j}\right)^{-1}}\right) \underbrace{}_{\substack{1 \leq i \leq m \\ 1 \leq j \leq n}} .
$$

Then, $X$ encodes the typical $\bullet$-entries, $Y$ encodes the typical $\times$-entries, and $Z$ encodes atypical entries.

Theorem 44. Let $L$ be a $K W$-module with special arc diagram (6-1). Then one has

$$
e^{\rho} R \cdot \operatorname{ch} L=(-1)^{r(t+q)}\left|\begin{array}{cc}
X & Z \\
0 & Y
\end{array}\right|
$$

Remark 45. The data defining (6-2) can also be determined from the standard arc diagram of a KW-module $L$. In particular, the exponents $a_{j}$ are the typical $\bullet$-entries, the exponents $b_{i}$ are the typical $\times$-entries, the exponent $z$ is the maximal atypical entry, $r$ is the number of arcs, $t$ is the number of typical $\bullet$-nodes below an arc, and $q$ is the number of typical $\times$-nodes below an arc. See Remark 23 to recall the relationship between the standard arc diagram and the special arc diagram.

Example 46. If the special arc diagram of $L$ is

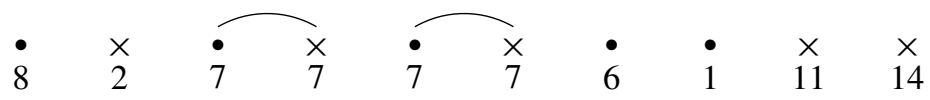


then

$e^{\rho} R \cdot \operatorname{ch} L$

$$
=-\left|\begin{array}{cccccccc}
x_{1}^{8} & x_{1}^{6} & x_{1} & \frac{x_{1}^{7} y_{1}^{7}}{1+\left(x_{1} y_{1}\right)^{-1}} & \frac{x_{1}^{7} y_{2}^{7}}{1+\left(x_{1} y_{2}\right)^{-1}} & \frac{x_{1}^{7} y_{3}^{7}}{1+\left(x_{1} y_{3}\right)^{-1}} & \frac{x_{1}^{7} y_{4}^{7}}{1+\left(x_{1} y_{4}\right)^{-1}} & \frac{x_{1}^{7} y_{5}^{7}}{1+\left(x_{1} y_{5}\right)^{-1}} \\
x_{2}^{8} & x_{2}^{6} & x_{2} & \frac{x_{2}^{7} y_{1}^{7}}{1+\left(x_{2} y_{1}\right)^{-1}} & \frac{x_{2}^{7} y_{2}^{7}}{1+\left(x_{2} y_{2}\right)^{-1}} & \frac{x_{2}^{7} y_{3}^{7}}{1+\left(x_{2} y_{3}\right)^{-1}} & \frac{x_{2}^{7} y_{4}^{7}}{1+\left(x_{2} y_{4}\right)^{-1}} & \frac{x_{2}^{7} y_{5}^{7}}{1+\left(x_{2} y_{5}\right)^{-1}} \\
x_{3}^{8} & x_{3}^{6} & x_{3} & \frac{x_{3}^{7} y_{1}^{7}}{1+\left(x_{3} y_{1}\right)^{-1}} & \frac{x_{3}^{7} y_{2}^{7}}{1+\left(x_{3} y_{2}\right)^{-1}} & \frac{x_{3}^{7} y_{3}^{7}}{1+\left(x_{3} y_{3}\right)^{-1}} & \frac{x_{3}^{7} y_{4}^{7}}{1+\left(x_{3} y_{4}\right)^{-1}} & \frac{x_{3}^{7} y_{5}^{7}}{1+\left(x_{3} y_{5}\right)^{-1}} \\
x_{4}^{8} & x_{4}^{6} & x_{4} & \frac{x_{4}^{7} y_{1}^{7}}{1+\left(x_{4} y_{1}\right)^{-1}} & \frac{x_{4}^{7} y_{2}^{7}}{1+\left(x_{4} y_{2}\right)^{-1}} & \frac{x_{4}^{7} y_{3}^{7}}{1+\left(x_{4} y_{3}\right)^{-1}} & \frac{x_{4}^{7} y_{4}^{7}}{1+\left(x_{4} y_{4}\right)^{-1}} & \frac{x_{4}^{7} y_{5}^{7}}{1+\left(x_{4} y_{5}\right)^{-1}} \\
x_{5}^{8} & x_{5}^{6} & x_{5} & \frac{x_{1}^{7} y_{1}^{7}}{1+\left(x_{5} y_{1}\right)^{-1}} & \frac{x_{1}^{7} y_{2}^{7}}{1+\left(x_{5} y_{2}\right)^{-1}} & \frac{x_{5}^{7} y_{3}^{7}}{1+\left(x_{5} y_{3}\right)^{-1}} & \frac{x_{5}^{7} y_{4}^{7}}{1+\left(x_{5} y_{4}\right)^{-1}} & \frac{x_{6}^{7} y_{5}^{7}}{1+\left(x_{6} y_{5}\right)^{-1}} \\
0 & 0 & 0 & y_{1}^{2} & y_{2}^{2} & y_{3}^{2} & y_{4}^{2} & y_{5}^{2} \\
0 & 0 & 0 & y_{1}^{11} & y_{2}^{11} & y_{3}^{11} & y_{4}^{11} & y_{5}^{11} \\
0 & 0 & 0 & y_{1}^{14} & y_{2}^{14} & y_{3}^{14} & y_{4}^{14} & y_{5}^{14}
\end{array}\right| .
$$

6.2. Two linear-algebraic lemmas. For the proof of Theorem 44, we will need the following lemmas. Suppose $m, n, r \in \mathbb{N}$ and $m, n \geqslant r$, and let $\mathcal{M}$ be the set of matrices of the form

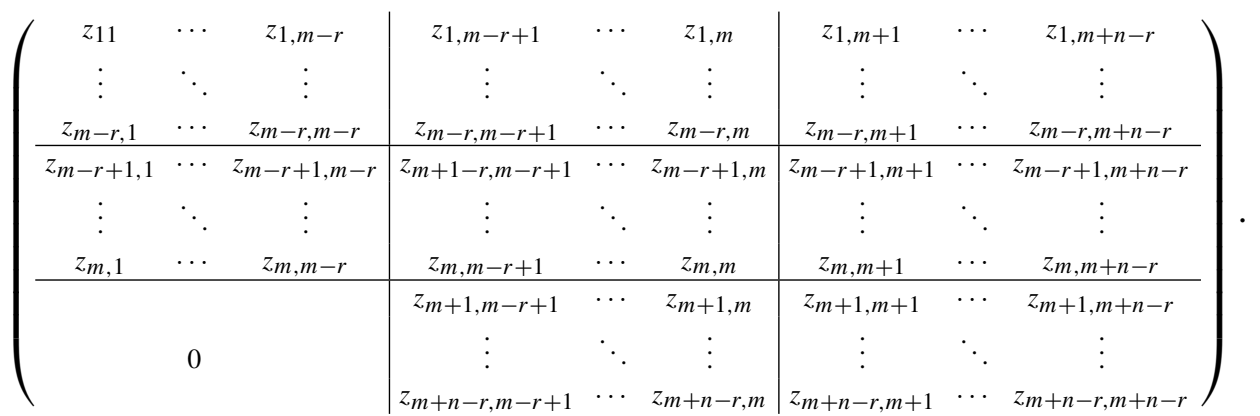

Lemma 47. There exists a unique polynomial $d\left(z_{i j}\right), d: M \rightarrow \mathbb{C}$ such that:

(1) $d$ is antisymmetric with respect to interchanges of the first $m$ rows and of the last $n$ columns.

(2) $d$ is linear with respect to row operations on the first $m$ rows and column operations on the last $n$ columns.

(3) $d$ specializes to 1 on all matrices of the form

$$
\left(\begin{array}{c|c|c}
I_{m-r} & 0 & Z \\
\hline 0 & I_{r} & 0 \\
\hline 0 & 0 & I_{n-r}
\end{array}\right),
$$


where I denotes the identity matrix and $Z$ is arbitrary.

Proof. The determinant satisfies all of the required conditions, proving existence. The proof of uniqueness is the same as the standard proof of the uniqueness of the determinant. Using row and column operations we can reduce any matrix in $\mathcal{M}$ to the form in condition (3).

Remark 48. Observe that a permutation of the first $m$ rows of a matrix $M \in M$ corresponds to a permutation of the subset $\{1, \ldots, m\}$ of the first indices of the elements $z_{i, j}$, and a permutation of the last $n$ columns of a matrix $M \in M$ corresponds to a permutation of the subset $\{m+1-r, \ldots, m+n-r\}$ of the second indices of the elements $z_{i, j}$.

Lemma 49. Let $M \in M$ be a matrix of the form (6-3). Then

$$
\operatorname{det} M=\frac{1}{r !} \sum_{w \in \operatorname{Sym}_{m} \times \operatorname{Sym}_{n}}(-1)^{l(w)} w\left(z_{1,1} z_{2,2} \ldots z_{m+n-r, m+n-r}\right),
$$

where $\operatorname{Sym}_{m}$ permutes the subset $\{1, \ldots, m\}$ of the first indices of the elements $z_{i, j}$ and $\operatorname{Sym}_{n}$ permutes the subset $\{m+1-r, \ldots, m+n-r\}$ of the second indices of the elements $z_{i, j}$.

Proof. By Lemma 47, we only need to check that the polynomial on the right-hand side satisfies the three conditions. The first two are clear. For the third, we need to count the number of elements $(u, v) \in \operatorname{Sym}_{m} \times \operatorname{Sym}_{n}$ that give a nonzero contribution to the alternating sum. It is easily seen that $\left.u\right|_{\{1, \ldots, m-r\}}$ and $\left.v\right|_{\{m+1, \ldots, m+n-r\}}$ must be identities. So to get a nonzero contribution (which must be 1) from the central block we need that $\left.u\right|_{\{m+1-r, \ldots, m\}}=\left.v\right|_{\{m+1-r, \ldots, m\}}$. These elements are all even, and there are $r$ ! of them.

6.3. Proof of Theorem 44. Let $L$ be a KW-module with special arc diagram (6-1). We define

$$
\begin{aligned}
t_{\lambda} & =\frac{e^{\lambda^{\rho}}}{\prod_{\beta \in S_{\lambda}}\left(1+e^{-\beta}\right)} \\
& =\prod_{i=1}^{p} x_{i}^{a_{i}} \prod_{i=p+1}^{m-r} x_{r+i}^{a_{i}} \prod_{k=1}^{r} \frac{\left(x_{p+k} y_{q+k}\right)^{z}}{1+\left(x_{p+k} y_{q+k}\right)^{-1}} \prod_{j=1}^{q} y_{j}^{b_{j}} \prod_{j=q+1}^{n-r} y_{r+j}^{b_{j}} .
\end{aligned}
$$

Then the Kac-Wakimoto character formula implies that

$$
e^{\rho} R \cdot \operatorname{ch} L=\frac{1}{r !} \sum_{w \in \operatorname{Sym}_{m} \times \operatorname{Sym}_{n}}(-1)^{l(w)} w\left(t_{\lambda}\right) .
$$


Now by applying Lemma 49 to the matrix in question we obtain

$$
\begin{aligned}
\left|\begin{array}{cc}
X & Z \\
0 & Y
\end{array}\right| & =\frac{1}{r !} \sum_{w \in \operatorname{Sym}_{m} \times \operatorname{Sym}_{n}}(-1)^{l(w)} w\left(\prod_{i=1}^{m-r} x_{i}^{a_{i}} \prod_{k=1}^{r} \frac{\left(x_{m-r+k} y_{k}\right)^{z}}{1+\left(x_{m-r+k} y_{k}\right)^{-1}} \prod_{j=1}^{n-r} y_{r+j} b_{r+j}\right) \\
& =\frac{1}{r !} \sum_{w \in \operatorname{Sym}_{m} \times \operatorname{Sym}_{n}}(-1)^{l(w)} w\left(\left(u^{-1}, v^{-1}\right) t_{\lambda}\right),
\end{aligned}
$$

where $u$ is the permutation sending $\left(x_{1}, \ldots, x_{m}\right)$ to

$$
\left(x_{1}, \ldots, x_{p}, x_{p+r+1}, \ldots, x_{m}, x_{p+1}, \ldots, x_{p+r}\right)
$$

and $v$ is the permutation sending $\left(y_{1}, \ldots, y_{n}\right)$ to

$$
\left(y_{q+1}, \ldots, y_{q+r}, y_{1}, \ldots, y_{q}, y_{q+r+1}, \ldots, y_{n}\right) \text {. }
$$

Then since $l(u)=r t$ and $l(v)=r q$, we have

$$
\left|\begin{array}{cc}
X & Z \\
0 & Y
\end{array}\right|=\frac{(-1)^{r(t+q)}}{r !} \sum_{w \in \operatorname{Sym}_{m} \times \operatorname{Sym}_{n}}(-1)^{l(w)} w\left(t_{\lambda}\right) .
$$

Combining (6-4) with (6-5) concludes the proof.

\section{Acknowledgments}

We would like to thank the following people for useful and interesting discussions: Jonah Blasiak, Ivan Dimitrov, Sergey Fomin, Maria Gorelik, Thomas Lam and Ricky Liu.

\section{References}

[Berele and Regev 1987] A. Berele and A. Regev, "Hook Young diagrams with applications to combinatorics and to representations of Lie superalgebras", Adv. in Math. 64:2 (1987), 118-175. MR 88i:20006 Zbl 0617.17002

[Bernstein and Leites 1980] I. N. Bernstein and D. A. Leites, "A formula for the characters of the irreducible finite-dimensional representations of Lie superalgebras of series Gl and sl", C. R. Acad. Bulgare Sci. 33:8 (1980), 1049-1051. In Russian. MR 82j:17020a

[Brundan 2003] J. Brundan, "Kazhdan-Lusztig polynomials and character formulae for the Lie superalgebra gl $(m \mid n)$ ”, J. Amer. Math. Soc. 16:1 (2003), 185-231. MR 2003k:17007 Zbl 1050.17004

[Brundan and Stroppel 2010] J. Brundan and C. Stroppel, "Highest weight categories arising from Khovanov's diagram algebra, II: Koszulity”, Transform. Groups 15:1 (2010), 1-45. MR 2011b:17014

[Brundan and Stroppel 2011] J. Brundan and C. Stroppel, "Highest weight categories arising from Khovanov's diagram algebra I: Cellularity”, Mosc. Math. J. 11:4 (2011), 685-722. MR 2918294 Zbl 1275.17012

[Brundan and Stroppel 2012] J. Brundan and C. Stroppel, "Highest weight categories arising from Khovanov's diagram algebra, IV: The general linear supergroup", J. Eur. Math. Soc. (JEMS) 14:2 (2012), 373-419. MR 2012m:17009 Zbl 1243.17004 
[Cheng et al. 2008a] S.-J. Cheng, J.-H. Kwon, and N. Lam, "A BGG-type resolution for tensor modules over general linear superalgebra", Lett. Math. Phys. 84:1 (2008), 75-87. MR 2009a:17009 Zbl 1172.17005

[Cheng et al. 2008b] S.-J. Cheng, W. Wang, and R. B. Zhang, "Super duality and KazhdanLusztig polynomials”, Trans. Amer. Math. Soc. 360:11 (2008), 5883-5924. MR 2009e:17008 Zbl 1234.17004

[Gorelik 2012] M. Gorelik, "Weyl denominator identity for finite-dimensional Lie superalgebras", pp. 167-188 in Highlights in Lie algebraic methods, edited by A. Joseph et al., Progr. Math. 295, Birkhäuser/Springer, New York, 2012. MR 2866851 Zbl 1292.17006

[Gorelik et al. 2012] M. Gorelik, V. G. Kac, P. Möseneder Frajria, and P. Papi, "Denominator identities for finite-dimensional Lie superalgebras and Howe duality for compact dual pairs", Jpn. J. Math. 7:1 (2012), 41-134. MR 2902248 Zbl 06132648

[Gruson and Serganova 2010] C. Gruson and V. Serganova, "Cohomology of generalized supergrassmannians and character formulae for basic classical Lie superalgebras", Proc. Lond. Math. Soc. (3) 101:3 (2010), 852-892. MR 2012a:17010 Zbl 1216.17005

[Van der Jeugt et al. 1990] J. Van der Jeugt, J. W. B. Hughes, R. C. King, and J. Thierry-Mieg, "Character formulas for irreducible modules of the Lie superalgebras $\operatorname{sl}(m / n)$ ", J. Math. Phys. 31:9 (1990), 2278-2304. MR 92b:17049 Zbl 0725.17004

[Kac 1977a] V. G. Kac, "Lie superalgebras", Advances in Math. 26:1 (1977), 8-96. MR 58 \#5803 Zbl 0366.17012

[Kac 1977b] V. G. Kac, "Characters of typical representations of classical Lie superalgebras", Comm. Algebra 5:8 (1977), 889-897. MR 56 \#3075 Zbl 0359.17010

[Kac 1978] V. Kac, "Representations of classical Lie superalgebras", pp. 597-626 in Differential geometrical methods in mathematical physics, II (University of Bonn, 1977), edited by K. Bleuler and A. Reetz, Lecture Notes in Math. 676, Springer, Berlin, 1978. MR 80f:17006 Zbl 0388.17002

[Kac and Wakimoto 1994] V. G. Kac and M. Wakimoto, "Integrable highest weight modules over affine superalgebras and number theory", pp. 415-456 in Lie theory and geometry, edited by J.-L. Brylinski et al., Progr. Math. 123, Birkhäuser, Boston, 1994. MR 96j:11056 Zbl 0854.17028

[Kac and Wakimoto 2014] V. G. Kac and M. Wakimoto, "Representations of affine superalgebras and mock theta functions", Transform. Groups 19:2 (2014), 383-455. MR 3200431 Zbl 06315917

[Moens and Van der Jeugt 2003] E. M. Moens and J. Van der Jeugt, "A determinantal formula for supersymmetric Schur polynomials”, J. Algebraic Combin. 17:3 (2003), 283-307. MR 2004f:05187 Zbl 1020.05070

[Moens and Van der Jeugt 2004] E. M. Moens and J. Van der Jeugt, "A character formula for atypical critical $\mathfrak{g l}(m \mid n)$ representations labelled by composite partitions", J. Phys. A 37:50 (2004), 12019-12039. MR 2005j:17008 Zbl 1077.17010

[Musson 2012] I. M. Musson, Lie superalgebras and enveloping algebras, Graduate Studies in Mathematics 131, American Mathematical Society, Providence, RI, 2012. MR 2906817 Zbl 1255.17001

[Musson and Serganova 2011] I. M. Musson and V. Serganova, "Combinatorics of character formulas for the Lie superalgebra $\mathfrak{g l}(m, n)$ ", Transform. Groups 16:2 (2011), 555-578. MR 2012e:17021 Zbl 1275.17018

[Serganova 1996] V. Serganova, "Kazhdan-Lusztig polynomials and character formula for the Lie superalgebra $\mathfrak{g l}(m \mid n)$ ”, Selecta Math. (N.S.) 2:4 (1996), 607-651. MR 98f:17007 Zbl 0881.17005

[Serganova 1998] V. Serganova, "Characters of irreducible representations of simple Lie superalgebras”, pp. 583-593 in Proceedings of the International Congress of Mathematicians, II (Berlin, 1998), Documenta Mathematica, Bielefeld, 1998. MR 99j:17010 Zbl 0898.17002 
[Serganova 2011] V. Serganova, "Kac-Moody superalgebras and integrability", pp. 169-218 in Developments and trends in infinite-dimensional Lie theory, edited by K.-H. Neeb and A. Pianzola, Progr. Math. 288, Birkhäuser, Boston, 2011. MR 2011m:17056 Zbl 1261.17025

[Sergeev 1984] A. N. Sergeev, "Tensor algebra of the identity representation as a module over the Lie superalgebras Gl $(n, m)$ and $Q(n)$ ", Mat. Sb. (N.S.) 123 (165):3 (1984), 422-430. In Russian; translated in Math. USSR 51 (1985), 419-427. MR 85h:17010

[Su and Zhang 2007] Y. Su and R. B. Zhang, "Character and dimension formulae for general linear superalgebra”, Adv. Math. 211:1 (2007), 1-33. MR 2008f:17012 Zbl 1166.17002

[Su and Zhang 2012] Y. Su and R. B. Zhang, "Generalised Jantzen filtration of Lie superalgebras I", J. Eur. Math. Soc. (JEMS) 14:4 (2012), 1103-1133. MR 2928846 Zbl 1295.17008

Communicated by Georgia Benkart

Received 2014-06-16 Revised 2015-02-17 Accepted 2015-03-28

mchmutov@umn.edu

Department of Mathematics, University of Minnesota, Minneapolis, MN 55455, United States

hoyt@tx.technion.ac.il

Department of Mathematics, Technion - Israel Institute of Technology, 3200003 Haifa, Israel

shifi@umich.edu

Department of Mathematics, University of Michigan, Ann Arbor, MI 48109, United States 


\section{Algebra \& Number Theory}

msp.org/ant

\section{EDITORS}

MANAGING EDITOR

Bjorn Poonen

Massachusetts Institute of Technology

Cambridge, USA

\author{
EDITORIAL BOARD CHAIR \\ David Eisenbud \\ University of California \\ Berkeley, USA
}

BOARD OF EDITORS

Georgia Benkart

Dave Benson

Richard E. Borcherds

John H. Coates

J-L. Colliot-Thélène

Brian D. Conrad

Hélène Esnault

Hubert Flenner

Sergey Fomin

Edward Frenkel

Andrew Granville

Joseph Gubeladze

Roger Heath-Brown

Craig Huneke

Kiran S. Kedlaya

János Kollár

Yuri Manin

Philippe Michel
University of Wisconsin, Madison, USA

University of Aberdeen, Scotland

University of California, Berkeley, USA

University of Cambridge, UK

CNRS, Université Paris-Sud, France

Stanford University, USA

Freie Universität Berlin, Germany

Ruhr-Universität, Germany

University of Michigan, USA

University of California, Berkeley, USA

Université de Montréal, Canada

San Francisco State University, USA

Oxford University, UK

University of Virginia, USA

Univ. of California, San Diego, USA

Princeton University, USA

Northwestern University, USA

École Polytechnique Fédérale de Lausanne
Susan Montgomery

Shigefumi Mori

Raman Parimala

Jonathan Pila

Anand Pillay

Victor Reiner

Peter Sarnak

Joseph H. Silverman

Michael Singer

Vasudevan Srinivas

J. Toby Stafford

Ravi Vakil

Michel van den Bergh

Marie-France Vignéras

Kei-Ichi Watanabe

Efim Zelmanov

Shou-Wu Zhang
University of Southern California, USA

RIMS, Kyoto University, Japan

Emory University, USA

University of Oxford, UK

University of Notre Dame, USA

University of Minnesota, USA

Princeton University, USA

Brown University, USA

North Carolina State University, USA

Tata Inst. of Fund. Research, India

University of Michigan, USA

Stanford University, USA

Hasselt University, Belgium

Université Paris VII, France

Nihon University, Japan

University of California, San Diego, USA

Princeton University, USA

\section{PRODUCTION}

production@msp.org

Silvio Levy, Scientific Editor

See inside back cover or msp.org/ant for submission instructions.

The subscription price for 2015 is US $\$ 255 /$ year for the electronic version, and $\$ 440 /$ year ( $+\$ 55$, if shipping outside the US) for print and electronic. Subscriptions, requests for back issues and changes of subscribers address should be sent to MSP.

Algebra \& Number Theory (ISSN 1944-7833 electronic, 1937-0652 printed) at Mathematical Sciences Publishers, 798 Evans Hall \#3840, c/o University of California, Berkeley, CA 94720-3840 is published continuously online. Periodical rate postage paid at Berkeley, CA 94704, and additional mailing offices.

ANT peer review and production are managed by EditFLOW ${ }^{\circledR}$ from MSP.

\section{PUBLISHED BY}

- mathematical sciences publishers

nonprofit scientific publishing

http://msp.org/

(C) 2015 Mathematical Sciences Publishers 


\section{Algebra \& Number Theory}

Volume $9 \quad$ No. $6 \quad 2015$

Bivariant algebraic cobordism

José LUIS GONZÁLEZ and KALLE KARU

Schubert decompositions for quiver Grassmannians of tree modules OLIVER LORSCHEID

Noncommutative geometry and Painlevé equations

ANDREI OKOUNKOV and ERIC RAINS

Electrical networks and Lie theory

ThOMas Lam and Pavlo PylyavskyY

The Kac-Wakimoto character formula for the general linear Lie superalgebra Michael Chmutov, Crystal Hoyt and ShifRa Reif

Effective Matsusaka's theorem for surfaces in characteristic $p$

Gabriele Di Cerbo and ANDREA FANELli

Adams operations and Galois structure

GEORGIOS PAPPAS 